Document downloaded from:

http://hdl.handle.net/10251/120297

This paper must be cited as:

Soto Francés, VM.; Pinazo Ojer, JM.; Sarabia Escrivà, EJ.; Martínez, PJ. (2019). On using the minimum energy dissipation to estimate the steady-state of a flow network and discussion about the resulting power-law:application to tree-shaped networks in HVAC systems. Energy. 172:181-195. https://doi.org/10.1016/j.energy.2019.01.060

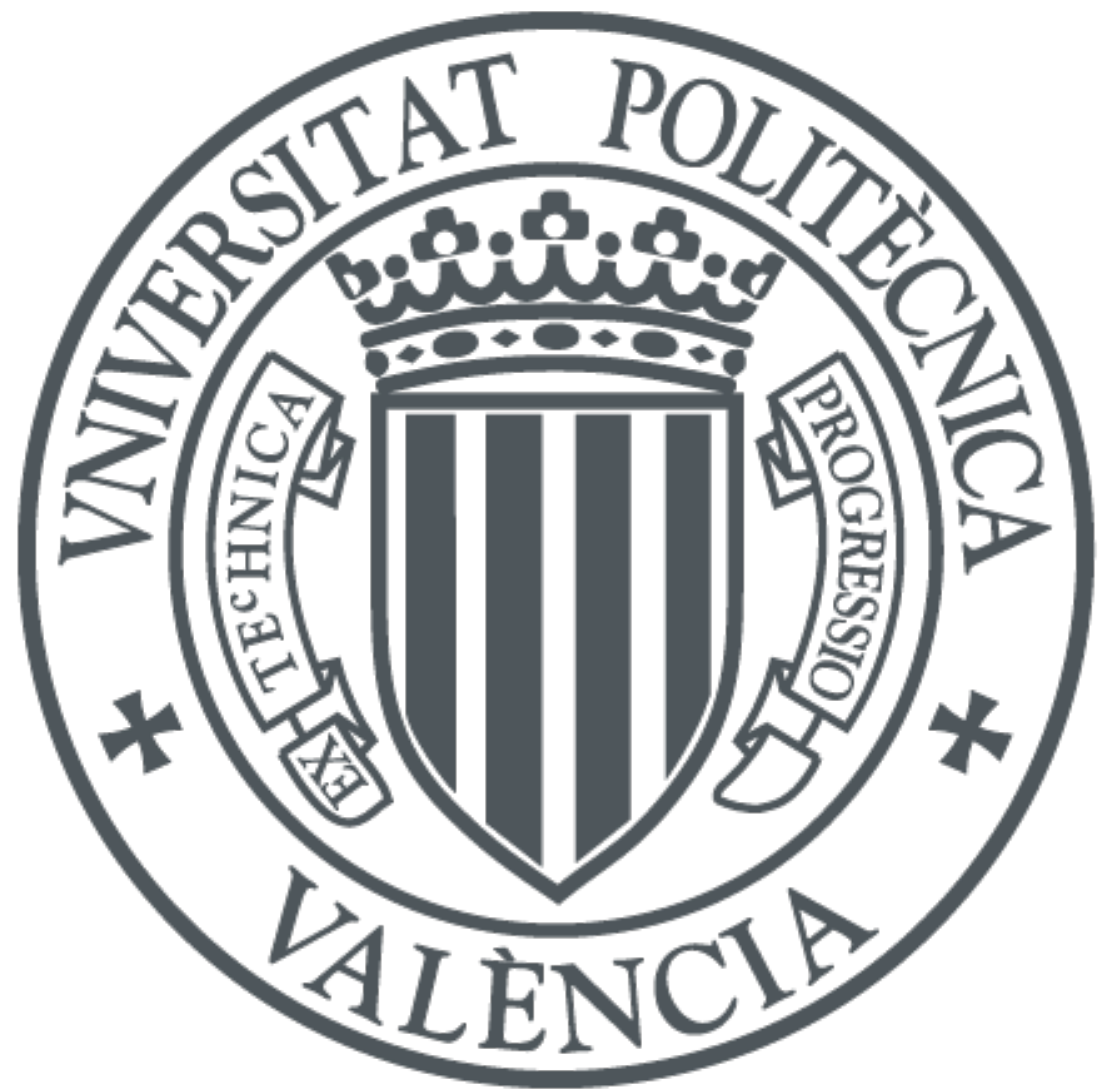

The final publication is available at

https://doi.org/10.1016/j.energy.2019.01.060

Copyright Elsevier

Additional Information 


\section{Accepted Manuscript}

On using the minimum energy dissipation to estimate the steady-state of a flow network and discussion about the resulting power-law:application to tree-shaped networks in HVAC systems

Víctor-Manuel Soto-Francés, José-Manuel Pinazo-Ojer, Emilio-José Sarabia-Escrivá,

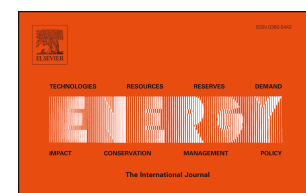
Pedro-Juan Martínez-Beltrán

PII:

$$
\text { S0360-5442(19)30062-3 }
$$

DOI:

$$
\text { https://doi.org/10.1016/j.energy.2019.01.060 }
$$

Reference: EGY 14533

To appear in: Energy

Received Date: 27 July 2018

Revised Date: 28 November 2018

Accepted Date: 13 January 2019

Please cite this article as: Soto-Francés Ví-Manuel, Pinazo-Ojer José-Manuel, Sarabia-Escrivá EmilioJosé, Martínez-Beltrán P-J, On using the minimum energy dissipation to estimate the steady-state of a flow network and discussion about the resulting power-law:application to tree-shaped networks in HVAC systems, Energy (2019), doi: https://doi.org/10.1016/j.energy.2019.01.060.

This is a PDF file of an unedited manuscript that has been accepted for publication. As a service to our customers we are providing this early version of the manuscript. The manuscript will undergo copyediting, typesetting, and review of the resulting proof before it is published in its final form. Please note that during the production process errors may be discovered which could affect the content, and all legal disclaimers that apply to the journal pertain. 


\title{
On using the minimum energy dissipation to estimate the steady-state of a flow network and discussion about the resulting power-law: application to tree-shaped networks in HVAC systems
}

\author{
Víctor-Manuel Soto-Francés ${ }^{\mathrm{a}, *}$, José-Manuel Pinazo-Ojer ${ }^{\mathrm{a},}$, Emilio-José \\ Sarabia-Escriváa ${ }^{\mathrm{a}}$, Pedro-Juan Martínez-Beltrán ${ }^{\mathrm{b}}$, \\ ${ }^{a}$ Departamento de Termodinámica Aplicada. ETSII. Universitat Politècnica de València. \\ C/Camino de Vera $s / n$ 46022 (Valencia) Spain \\ ${ }^{b}$ Departamento de Ingeniería Mecánica y Energía. Universidad Miguel Hernández. Avda. \\ de la Universidad s/n 03202,(Elche) Spain
}

\section{Abstract}

The paper analyses how to compute the steady-state flow distribution through a given network by using the Minimum Entropy Production (MinEP) principle. For isothermal and incompressible flows, this is equivalent to the minimal dissipation of energy. The conditions that make this method equivalent to the conventional one are studied. There must exist a power-law for the energy dissipation (entropy generation) where the exponent must be the same for the whole network. To our knowledge, Niven was the first to get to this result. However he applied MinEP only to parallel pipes and unfortunately discarded it as a general method.

The paper shows why it cannot be discarded yet. We discuss the role of the chosen exponent $m$ and its link to the underlying physical phenomena.

Moreover it is shown that there is a "hidden" fixed point value problem that must be studied further. The method introduced in this paper is developed specially for tree-shaped duct-networks which are frequently encountered

\footnotetext{
${ }^{*}$ Corresponding author

Email addresses: vsoto@ter.upv.es, Tel.:+34 3879326 Fax:+34 3877329 (Víctor-Manuel Soto-Francés ), jmpinazo@ter.upv.es (José-Manuel Pinazo-Ojer ), emsaes@upvnet.upv.es (Emilio-José Sarabia-Escrivá ), pjuan.martinez@umh.es (Pedro-Juan Martínez-Beltrán )
} 
in HVAC (Heating Ventilation and Air Conditioning) systems. The paper explains briefly what triggered this research; specifically, difficulties related with branched junctions, mainly in return-type networks.

Finally, we illustrate the method with some examples.

Keywords: MinEP, Entropy generation, steady-state, flow network, HVAC duct network

\section{Introduction}

The importance of energy efficiency related to environmental issues increases every year. In HVAC (Heating Ventilation and Air Conditioning) facilities, the distribution of fluids to transfer thermal power is widespread. A better design and analysis of the correct air flow distribution through the network is crucial to achieve a good performance of the whole system (see for instance [1).

However, a better efficiency is also related to the concept of entropy. Efforts have been done in order to bring it to daily engineering practice. Remarkable are those from A. Bejan et al. ([2, [3]), and many others after him. More recent efforts like those from Herwig H. [4] in 2012 try to boost the attention to its application to fundamental fluid and heat flow problems. In [5] Tammo, Herwig et al. proposes the entropic potential number which is related to the idea of losses (loss of availability) and the entropy generation, as a measure of those losses. Moreover in [6] Herwig and Schmandt proposed to link the entropy generation to a very well-known concept in fluid mechanics; the head loss coefficient. This idea of a loss coefficient seems to have attracted others researchers $([7])$.

The minimisation of the entropy generation is not used here as a constructal law (see [8]) where the unknown is the geometry (see [9]). In other words, the purpose of the method is not the design of duct-networks. Here we have used the principle of minimum entropy generation or according to our hypotheses, minimum energy dissipation, as a means to discover the steady-state flow distribution through an already existing network. However this does not mean that 
it cannot be used to design networks but this needs further research. Therefore we stress the fact that our aim is the analysis of an existing network, not its sizing or geometrical design.

By energy dissipation we mean the conversion of mechanical energy into internal energy.

This paper goes closer to the ideas of R. Niven [10] which can be summarised in the sentence: "the study of extremum methods to discover the steady-state of systems". In [10] Niven deals with two principles; MinEP (Minimum Entropy Production) with respect the flow distribution and MaxEP (Maximum Entropy Production) with respect to the choice of the flow regime.

This paper extends Niven's results [10] to tree-shaped networks (although we think they can even be extended to general ones). However, something new comes up. We show that, in the most general situation, there exists a fixed point value problem associated with the steady-state flow distribution through the network. Moreover, any fixed point must also be a solution of the conventional method (based on energy and mass balances). The solutions obtained by either method are the same. However, we gain some additional insight which poses some questions; what is the nature of the fixed point?. Is it a minimum or a maximum of the net dissipation function?. What are the conditions which guarantee that there is just one fixed point (a unique solution)?. Recall the fact that any conventional solution must also be a fixed point in our method and vice versa. Daily engineering practice (at least in the HVAC field) suggests that there must be just a single fixed point (i.e. a unique solution). In other words, there are no reports in the literature about networks switching back and forth among different steady-states. In all cases found the fixed point is a minimum of the network dissipation function.

Finally we would like to make some additional comments to this introduction. As Niven mentions in [10] :"...the term steady-state is something of a misnomer, since it refers only to a constant mean flow ...". Niven also proposes deeper and interesting ideas about the use of the entropy concept. In [1] he proposes the idea of maximising, not the thermodynamic entropy, but 
an information entropy of the system flows (flux entropy) for the analysis of its steady-state. In the literature it is known as the the MaxEnt principle. Niven deduces from it a relationship with the thermodynamic entropy generation. MaxEnt applied to the flux entropy would include, according to him, other principles like; the MinEP (minimum entropy generation) and MaxEP (maximum entropy generation) as particular cases.

We have tried to discover the usefulness of the method without going into such details but it is referenced here for the interested reader.

\subsection{Motivation}

This short subsection adds extra content. It explains briefly what triggered this research. It was motivated by some difficulties found mainly when designing return duct-networks. The source of the difficulties came from branched junctions. In return HVAC duct-networks, there exists the possibility of getting negative head loss coefficients in the branched junctions (see the physical explanation in [12]). The fact that the generated entropy or the energy dissipated is always a positive quantity, stimulated this research.

The interaction between the branches of a junction is usually neglected in networks with very long pipes/ducts but it is not the case for HVAC air ductnetworks.

Roughly stated the difficulty was the following. In order to find the operating point of a net there exists a very common or popular method. It is based on intersecting the fan curve with the network system characteristic curve. This network curve is obtained, in turn, by compressing the network by using two equivalent duct sections; one for sections arranged in series and another for sections in Y (series+parallel). The method is, in fact, iterative since after an operating point is estimated, the net must be decompressed to re-calculate both the flows and the respective new hydraulic resistances. The expression of the equivalent resistance, for the $\mathrm{Y}$ case, contains a square root of the hydraulic resistances at the parallel branches. This square root along with a bad initial guess during iteration and the possibility of achieving a negative head loss in 
branched junctions was the origin of the difficulties. This does not mean that there is no solution. It does have one, however this negative head loss obliged us to devise a special search algorithm.

The detailed explanation is deferred to another paper when dealing with branched juctions. This paper is just a first step and focuses on exposing the main new ideas.

Last but not least, despite the reason that triggered the research, the method usefulness could be wider.

\section{Analysis of losses in a conduit component in steady-state}

The aim of this section is to relate the concept of loss to the entropy generation and to the energy dissipation.

The energy balance equation, in the steady-state, can be written as (see 13 [14] or [15]):

$$
\dot{m}\left[\left(u_{2}+\frac{\alpha_{2} \bar{v}_{2}^{2}}{2}+g z_{2}\right)-\left(u_{1}+\frac{\alpha_{1} \bar{v}_{1}^{2}}{2}+g z_{1}\right)\right]=\dot{Q}_{12}-\dot{W}_{12}
$$

with state variables on the left and action variables on the right (see figure (1)) and $\alpha \approx 1$ for turbulent regime while in laminar regime $\alpha=2$ [17.

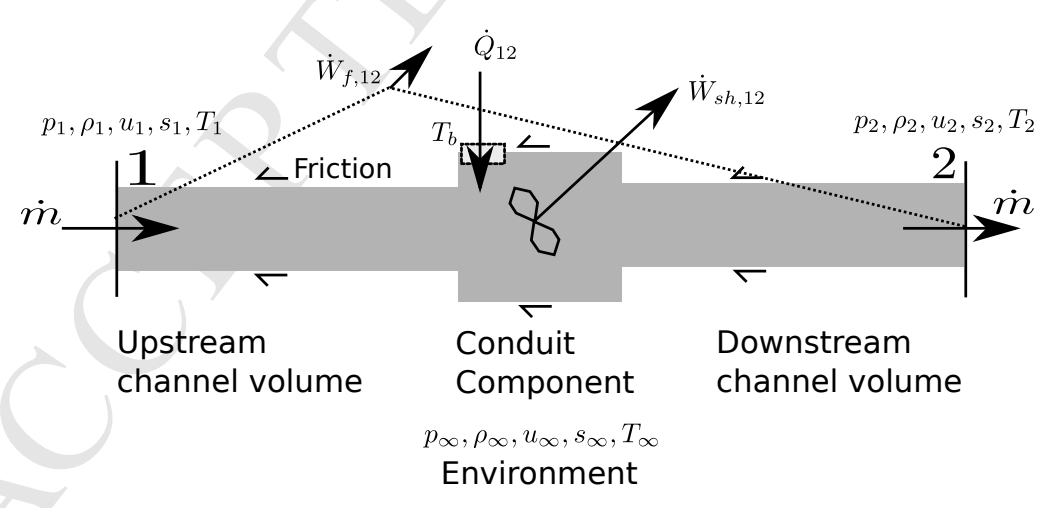

Figure 1: Fluid flow inside a conduit component.(Adapted from [16]). 
Dividing equaqtion (1) by $\dot{m}$, we get:

$$
\left(u_{2}-u_{1}\right)+\frac{\left(\alpha_{2} \bar{v}_{2}^{2}-\alpha_{1} \bar{v}_{1}^{2}\right)}{2}+g\left(z_{2}-z_{1}\right)=q_{12}-\left(w_{s h, 12}+w_{f, 12}\right)
$$

If the fluid flow is assumed to be incompressible then the internal energy $u$ changes because of two effects; the heat transfer $q_{12}$ or the energy dissipation, that is, the conversion of mechanical energy into internal energy. In order to make such dissipation "visible", equation 2 can be split into two as (see [15]):

$$
\begin{aligned}
& \text { mechanical: } \frac{\left(\alpha_{2} \bar{v}_{2}^{2}-\alpha_{1} \bar{v}_{1}^{2}\right)}{2}+g\left(z_{2}-z_{1}\right)=-\left(w_{s h, 12}+w_{f, 12}\right)-\varphi_{12} \\
& \text { thermal: }\left(u_{2}-u_{1}\right)=q_{12}+\varphi_{12} \\
& \text { or, using } w_{f, 12}=\left(p_{2} / \rho_{2}-p_{1} / \rho_{1}\right) \text { and } \rho_{1}=\rho_{2}=\rho: \\
& \text { mechanical: }\left(\frac{p_{2}}{\rho}-\frac{p_{1}}{\rho}\right)+\frac{\left(\alpha_{2} \bar{v}_{2}^{2}-\alpha_{1} \bar{v}_{1}^{2}\right)}{2}+g\left(z_{2}-z_{1}\right)=-w_{s h, 12}-\varphi_{12} \\
& \text { thermal: }\left(u_{2}-u_{1}\right)=q_{12}+\varphi_{12}
\end{aligned}
$$

The mechanical part (left side) is the well-known Bernouilli equation while the right hand side contains a term like:

$$
\varphi=\frac{\dot{\Phi}}{\dot{m}}
$$

which is the mechanical energy dissipation into internal energy per unit of mass. If we assume that the flow is isothermal and at the same temperature than the environment, i.e., $T_{\infty}=T_{b}=T_{\text {fluid }}$, then it can be related to the entropy generation (see [13]):

$$
\begin{aligned}
& \text { per unit mass: } \varphi=T \dot{S}_{g e n} / \dot{m}=T s_{g e n} \\
& \text { per unit of time: } \dot{\Phi}=T \dot{S}_{g e n}
\end{aligned}
$$

In other words, it is assumed that the only/main source of entropy generation is the conversion of mechanical energy into internal energy. 
In [18 Herwig proposed to relate the dissipation inside straight pipes to the Darcy-Weisbach friction factor $f_{D}$ as:

$$
\varphi_{12}=\underbrace{f_{D, 12} \frac{L_{12}}{D_{h}}}_{K_{p}} \cdot \frac{\bar{v}^{2}}{2}
$$

or in differential form:

$$
\frac{d \varphi}{d x}=f_{D} \frac{1}{D_{h}} \frac{\bar{v}^{2}}{2}
$$

For fully developed flow in horizontal pipes $\left(z_{2}=z_{1}\right)$, without changes in kinetic energy $\left(\alpha_{2} \bar{v}_{2}^{2}=\alpha_{1} \bar{v}_{1}^{2}\right)$ and $w_{s h}=0$, equation (8) (using eq.4a) can be written in terms of pressure $p$ as:

$$
f_{D}=\frac{d \varphi}{d x} \frac{2 D_{h}}{\bar{v}^{2}}=-\frac{d p}{d x} \frac{2 D_{h}}{\rho \bar{v}^{2}}=\frac{8 \tau_{w}}{\rho \bar{v}^{2}}
$$

or after integrating it:

$$
\varphi_{12}=f_{D, 12} \frac{L_{12}}{D_{h}} \frac{\bar{v}^{2}}{2}=-\frac{\Delta p_{12}}{\rho}=\frac{\Delta p_{21}}{\rho}
$$

which corresponds, although written differently, with equation (3.12) in [2]. Therefore, for an incompressible isothermal flow at the same temperature as the ambient $\Delta p_{21}=\varphi_{12} \cdot \rho=T_{\infty} \cdot s_{\text {gen }} \cdot \rho=w_{\text {lost }} \cdot \rho$, the measured pressure drop is related to the mechanical energy dissipated into internal energy (heat) per unit of volume and is proportional to the entropy generated and the exergy (availability) lost.

For any other type of conduit element a similar definition to $(7)$ is commonly used (see [19]):

$$
\varphi=K_{\varphi} \cdot \frac{\bar{v}^{2}}{2}
$$

Finally, the analysis of the losses could be extended to other types of flows, see for instance [20] and [19]. There, Herwig generalises the idea of a loss coefficient by defining an exergy loss coefficient $K_{E}$. For our purposes, the hypotheses made are enough. 


\section{Using the Minimum entropy generation principle for estimating the steady-state flow distribution in a tree-shaped network.}

The aim of this section is to discuss, under what conditions, the traditional methods of pipe network analysis are equivalent to the use of the minimum entropy production (MinEP) principle or according to our aforementioned hypotheses to the minimum energy dissipation. Such equivalence, hopefully, would allow us to turn, the network analysis problem, into a minimisation problem.

Our concrete goal is to estimate what is the steady-state air flow distribution in a tree-shaped duct network when its total input/output flow $\dot{V}_{T}$ is pre-defined or fixed (see figure (3)). Firstly, we review the conventional formulation and secondly we will search for the conditions that guarantee that the MinEP is equivalent to that formulation.

\subsection{Traditional closed-form solutions}

Conventionally, the closed-form solution to this problem is obtained based on energy and mass balances (see [21] or a recent review [22]):

- Mechanical energy conservation. The energy must be conserved between any two points of the network. This fact can be stated in different forms. Along any path from node $A$ to $B$, the total specific energy difference must be equal. This specific energy can be measured in different ways and accordingly receives different names. The specific mechanical energy $e_{m}$ per unit of fluid weight $\gamma=g \rho$ is named head $H$ (see eq. 12). A closed loop is any path that starts and ends at the same node $A$. The energy change around any closed loop must be zero. Finally there is a special loop named pseudo-loop. It is a path between two nodes $A$ and $B$ of known specific energy levels such as two tanks or reservoirs (like a big room in HVAC). In this case, the energy change must be equal to a fixed known value (for instance expressed in head: $H_{A}-H_{B}=$ constant). Note that both nodes can be the same, in which case, the closed loop case is recovered. 
The relationship between the mechanical energy per unit mass $\left(e_{m}\right)$, per unit of weight (head $H$ ) and per unit of volume (total pressure $p_{T}$ ) is:

$$
\begin{aligned}
e_{m} & =\frac{p}{\rho}+\frac{\alpha \bar{v}^{2}}{2}+g z \\
H=\frac{e_{m}}{g} & =\frac{p}{g \rho}+\frac{\alpha \bar{v}^{2}}{2 g}+z \\
p_{T}=e_{m} \cdot \rho & =p+\frac{\rho \alpha \bar{v}^{2}}{2}+\rho g z
\end{aligned}
$$

Let us see exactly how this energy balance looks like in a loop. By assuming that in any section the shaft work $w_{s h}=0$, the energy balance equation 4a, applied to the loop of figure (2) has the form shown in equation (13).

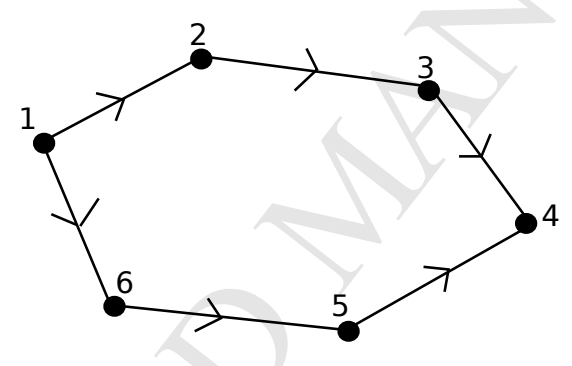

Figure 2: Flow loop. Arrows indicate the assumed flow sense.

In words, it means that the sum of the mechanical energy losses within the loop must be zero. Looking at figure (2), the losses from node 1 to node 4 along the path $1-2-3-4$ must be equal to the losses along the 
other path $1-6-5-4$.

$$
\begin{gathered}
e_{m, 1}-e_{m, 2}=\varphi_{12} \\
+e_{m, 2}-e_{m, 3}=\varphi_{23} \\
+e_{m, 3}-e_{m, 4}=\varphi_{34} \\
+e_{m, 4}-e_{m, 5}=-\varphi_{54} \\
+e_{m, 5}-e_{m, 6}=-\varphi_{65} \\
+e_{m, 6}-e_{m, 1}=-\varphi_{16}
\end{gathered}
$$

$$
0=\varphi_{12}+\varphi_{23}+\varphi_{34}-\left(\varphi_{54}+\varphi_{65}+\varphi_{16}\right)
$$

- Mass conservation or continuity. The sum of the ingoing mass flow rates of a node must be equal to the sum of its outgoing mass flow rates.

The unknowns in the steady-state analysis are the flows $\dot{V}_{j}$ in each $j$-section and the specific energy at each junction node. Thus, in a system with nnode junction nodes and nsect sections the number of unknowns is nnode + nsect.

\subsection{Minimisation of the dissipation}

In this section it is deduced and proved, mathematically, under what conditions the minimisation of the dissipated energy is equivalent to the previous conventional method. Stated in another way, the minimisation of the energy dissipated by the network will allow us to find how a given (forced) flow rate $\dot{V}_{T}$ at one section is distributed through an already sized duct-network. Notice that the $\dot{V}_{T}$ sense can be either an inlet (supply-network) or outlet flow (returnnetwork). The energy dissipated by any network can be obtained simply by adding the dissipation at each $j$ section as:

$$
\dot{\Phi}=\sum_{j=1}^{n s e c t}\left(\varphi_{j} \cdot \rho\right) \cdot \dot{V}_{j}
$$


By choosing a certain fixed volume flow rate $\dot{V}_{T}$, the previous equation can be expressed as:

$$
\dot{\Phi}=\left(\varphi_{T} \cdot \rho\right) \cdot \dot{V}_{T}=\sum_{j=1}^{n s e c t}\left(\varphi_{j} \cdot \rho\right) \cdot \dot{V}_{j}=\sum_{j=1}^{n s e c t} \widehat{\varphi}_{j} \cdot \dot{V}_{j}=\widehat{\varphi}_{T} \cdot \dot{V}_{T}
$$

where by $\widehat{\varphi}=\varphi \cdot \rho$ the dissipation per unit of volume is meant. 1

If we divide by $\dot{V}_{T}$ then:

$$
F\left(\psi_{1}, \psi_{2}, \ldots, \psi_{n s e c t}\right)=\frac{\dot{\Phi}}{\dot{V}_{T}}=\widehat{\varphi}_{T}=\sum_{j=1}^{n s e c t} \widehat{\varphi}_{j} \cdot \psi_{j}
$$

The $\psi_{j}=\dot{V}_{j} / \dot{V}_{T}$ are the volume flow rate ratios. They can be arranged in vector form as $\vec{\psi}=\left(\psi_{1}, \psi_{2}, \ldots, \psi_{n s e c t}\right)^{T}$ and represents the distribution of the $\dot{V}_{T}$ through the network.

Figure 3 shows a general tree-shaped network, typical of HVAC systems. It can be either a supply or a return network ( $\dot{V}_{T}$ sense would be reversed). For a supply network, point $B$ represents the air at the room and therefore its kinetic energy is assumed to be zero and point $A$ is the fan outlet. Notice that in this case $\widehat{\varphi}_{T}$ in equation (16), equals the total pressure difference between points $A$ and $B$ :

$$
\widehat{\varphi}_{T}=\Delta p_{T}
$$

The flow ratio $\psi_{i}$ at leaf $i$ of the tree receives a special name: $x_{i}=\dot{V}_{i} / \dot{V}_{T}$. These have been chosen as the flows in the pseudo-loops because they are meaningful in HVAC systems as they are the targeted or desired supply/return flows. Recall that our aim is to discover the flow distribution through a given network not its geometrical design, or in other words, how close are the actual flows from these targeted flows. The flow ratio $\psi_{j}$ at each $j$ section can be expressed as a function of these $x_{i}$. They can be included inside equation (16) by noticing

\footnotetext{
${ }^{1}$ Notice that for the case of a horizontal pipe $\widehat{\varphi}=\Delta p$, i.e., equals the pressure drop
} 


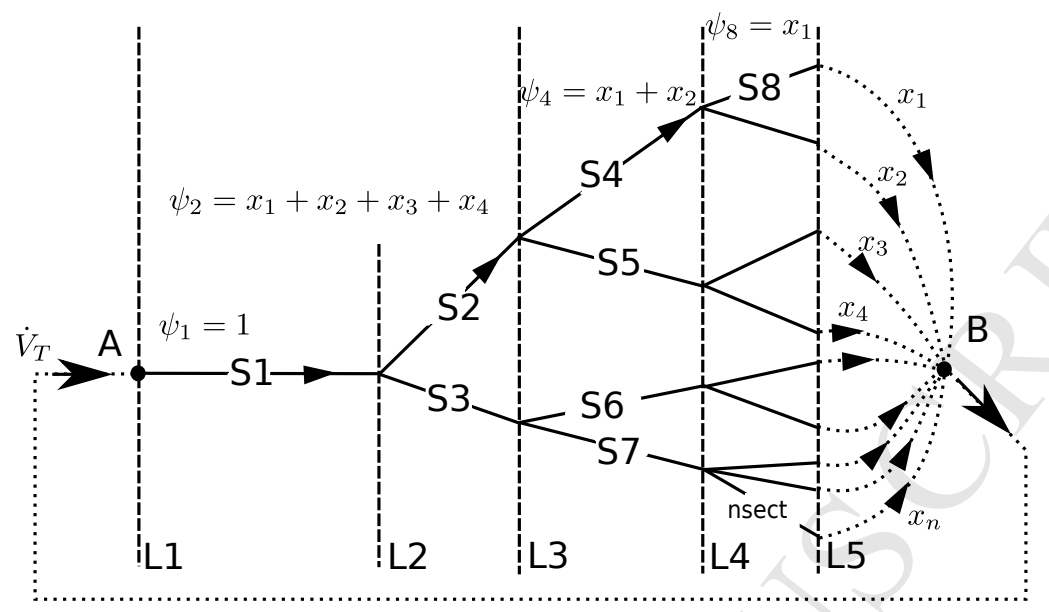

Figure 3: General tree-shaped duct network with 5 levels and nsect sections. (Dotted lines represent pseudo-loops.)

that the dependency of $\psi_{j}$ on $x_{i}$ is simply (see equation (18)).

$$
\psi_{j}=\sum_{\text {if leaf } i \text { is downstream } j} x_{i}
$$

Notice that not all the $x_{i}$ are independent since one flow rate $\dot{V}_{T}$ is enforced and the mass conservation constraint must be fulfilled. Therefore if an $x_{i}$ is arbitrarily chosen $\left(x_{n}\right.$, for instance) then the following equation holds;

$$
x_{n}=1-\sum_{i=1}^{n-1} x_{i}
$$

Thus the components of the vector $\vec{x}=\left(x_{1}, x_{2}, \ldots, x_{n-1}\right)^{T}$ are actually the independent variables.

Summarizing, a general mapping $g$ can be defined from an independent set of flow ratios $\vec{x}$ to the flow at the sections $\vec{\psi}$, as:

$$
g: \vec{x} \in \mathbb{R}^{n-1} \rightarrow \vec{\psi} \in \mathbb{R}^{n s e c t}
$$

which allows us to rewrite equation (16), by using the map composition with 
$g$, as:

$$
(F \circ g)\left(x_{1}, x_{2}, \ldots, x_{n-1}\right)=\sum_{j=1}^{n s e c t} \widehat{\varphi}_{j} \cdot \psi_{j}(\vec{x})
$$

In concrete for a tree-shaped network the $g$ map can be written as:

$$
\vec{\psi}(\vec{x}): \quad \vec{\psi}_{n \text { sect } \times 1}=[M]_{n s e c t \times n} \cdot\left([v 1]_{n \times 1}+[B]_{n \times(n-1)} \cdot \vec{x}_{(n-1) \times 1}\right)
$$

How to construct the matrices $[M],[v 1]$ and $[B]$ is best illustrated, in what follows, by using the example network of figure (4). Each row of matrix $[M]$ represents the equation (18), its entries are just 0 and 1 , therefore for this example:

$$
[M]=\left[\begin{array}{llll}
1 & 1 & 1 & 1 \\
1 & 1 & 0 & 0 \\
0 & 0 & 1 & 1 \\
1 & 0 & 0 & 0 \\
0 & 1 & 0 & 0 \\
0 & 0 & 1 & 0 \\
0 & 0 & 0 & 1
\end{array}\right]
$$

The term between brackets in equation (22) maps $\vec{x}$ to $\left(x_{1}, x_{2}, \ldots, x_{n}\right)$, therefore the last row is equal to equation $(19)$. For this example:

$$
[v 1]+[B] \cdot \vec{x}=\left[\begin{array}{c}
0 \\
0 \\
0 \\
1
\end{array}\right]+\left[\begin{array}{ccc}
1 & 0 & 0 \\
0 & 1 & 0 \\
0 & 0 & 1 \\
-1 & -1 & -1
\end{array}\right] \cdot\left[\begin{array}{c}
x_{1} \\
x_{2} \\
x_{3}
\end{array}\right]
$$

Since we look for the conditions that minimise the equation (21) we need to evaluate its first and second derivatives.

The derivative $\mathbb{D} g \equiv\left[\psi^{\prime}\right]$ (Jacobian) of the flow distribution vector $\vec{\psi}(\vec{x})$ with respect to the $\vec{x}$ can be obtained easily. A mnemonic rule can be devised. Take $[M]^{T}$ select the row of the $x_{i}$ previously selected to be removed ( $x_{n}$ in our case), multiply it by -1 and add that row to the others. Finally remove it (row $n$ in our case) from the matrix. 


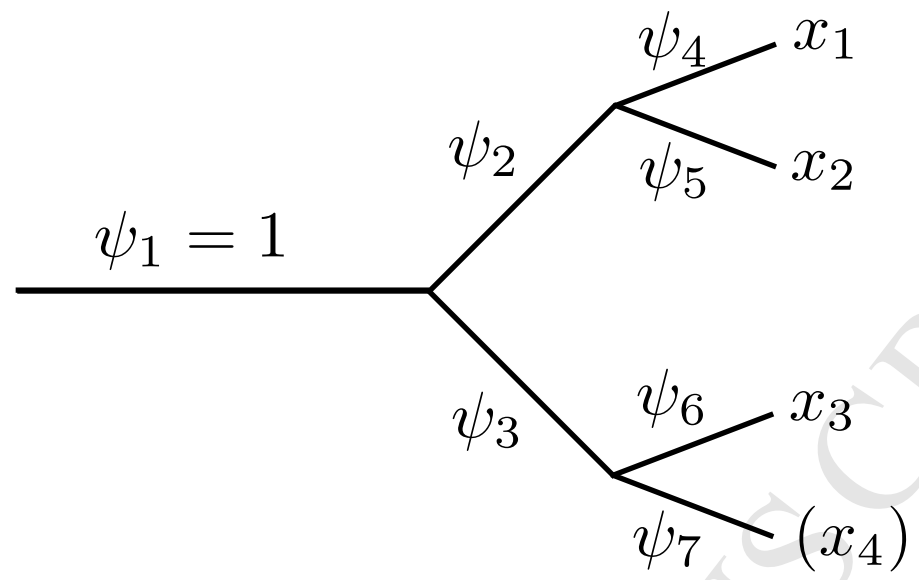

Figure 4: Example of a tree-shaped duct network $($ nsect $=7)$.

The result is $\left[\psi^{\prime}\right]^{T}$. In the case of the example (see figure 44 ) the matrix is:

$\left[\psi^{\prime}\right]^{T}=\left[\begin{array}{lllllll}\frac{\partial \psi_{1}}{\partial x_{1}} & \frac{\partial \psi_{2}}{\partial x_{1}} & \frac{\partial \psi_{3}}{\partial x_{1}} & \frac{\partial \psi_{4}}{\partial x_{1}} & \frac{\partial \psi_{5}}{\partial x_{1}} & \frac{\partial \psi_{6}}{\partial x_{1}} & \frac{\partial \psi_{7}}{\partial x_{1}} \\ \frac{\partial \psi_{1}}{\partial x_{2}} & \frac{\partial \psi_{2}}{\partial x_{2}} & \frac{\partial \psi_{3}}{\partial x_{2}} & \frac{\partial \psi_{4}}{\partial x_{2}} & \frac{\partial \psi_{5}}{\partial x_{2}} & \frac{\partial \psi_{6}}{\partial x_{2}} & \frac{\partial \psi_{7}}{\partial x_{2}} \\ \frac{\partial \psi_{1}}{\partial x_{3}} & \frac{\partial \psi_{2}}{\partial x_{3}} & \frac{\partial \psi_{3}}{\partial x_{3}} & \frac{\partial \psi_{4}}{\partial x_{3}} & \frac{\partial \psi_{5}}{\partial x_{3}} & \frac{\partial \psi_{6}}{\partial x_{3}} & \frac{\partial \psi_{7}}{\partial x_{3}}\end{array}\right]=\left[\begin{array}{ccccccr}0 & 1 & -1 & 1 & 0 & 0 & -1 \\ 0 & 1 & -1 & 0 & 1 & 0 & -1 \\ 0 & 0 & 0 & 0 & 0 & 1 & -1\end{array}\right]$

If the vectors are arranged in column form, the stationarity condition of the energy dissipation (see equation (21)) implies that:

$$
\mathbb{D} F(\vec{\psi}(\vec{x}))=\mathbb{D}(F \circ g)=\mathbb{D}\left(\left[\begin{array}{c}
\widehat{\varphi}_{1} \\
\widehat{\varphi}_{2} \\
\ldots \\
\widehat{\varphi}_{\text {nsect }}
\end{array}\right]^{T} \cdot\left[\begin{array}{c}
\psi_{1} \\
\psi_{2} \\
\cdots \\
\psi_{\text {nsect }}
\end{array}\right]\right)=\overrightarrow{0}
$$

The total derivative of the dissipation function with respect to $\vec{x}$ gives:

$$
\left[\begin{array}{c}
0 \\
0 \\
\cdots \\
0
\end{array}\right]=\left[\begin{array}{cccc}
\frac{\partial \widehat{\varphi}_{1}}{\partial x_{1}} & \frac{\partial \widehat{\varphi}_{2}}{\partial x_{1}} & \ldots & \frac{\partial \widehat{\varphi}_{n \text { duct }}}{\partial x_{1}} \\
\frac{\partial \widehat{\varphi}_{1}}{\partial x_{2}} & \frac{\partial \widehat{\varphi}_{2}}{\partial x_{2}} & \ldots & \frac{\partial \widehat{\varphi}_{\text {nduct }}}{\partial x_{2}} \\
\ldots & \ldots & \ldots & \\
\frac{\partial \widehat{\varphi}_{1}}{\partial x_{n-1}} & \frac{\partial \widehat{\varphi}_{2}}{\partial x_{n-1}} & \ldots & \frac{\partial \widehat{\varphi}_{n \text { duct }}}{\partial x_{n-1}}
\end{array}\right] \cdot\left[\begin{array}{c}
\psi_{1} \\
\psi_{2} \\
\ldots \\
\psi_{\text {nduct }}
\end{array}\right]+\left[\psi^{\prime}\right]^{T} \cdot\left[\begin{array}{c}
\widehat{\varphi}_{1} \\
\widehat{\varphi}_{2} \\
\ldots \\
\widehat{\varphi}_{\text {nduct }}
\end{array}\right]
$$


However, as it will be clear afterwards, it is much more useful to use the mapping $g$ (see equation 20 ) and to apply the chain rule (see [23]):

$$
\mathbb{D}(F \circ g)=(\mathbb{D} F \circ g) \cdot \mathbb{D} g
$$

and therefore equation (27) can be written as:

$$
\begin{gathered}
\mathbb{D}(F \circ g)^{T}=\left[\begin{array}{c}
0 \\
0 \\
\ldots \\
0
\end{array}\right]= \\
=\left[\psi^{\prime}\right]^{T} \cdot\left(\left[\begin{array}{cccc}
\frac{\partial \widehat{\varphi}_{1}}{\partial \psi_{1}} & \frac{\partial \widehat{\varphi}_{2}}{\partial \psi_{1}} & \ldots & \frac{\partial \widehat{\varphi}_{\text {nduct }}}{\partial \psi_{1}} \\
\frac{\partial \widehat{\varphi}_{1}}{\partial \psi_{2}} & \frac{\partial \widehat{\varphi}_{2}}{\partial \psi_{2}} & \ldots & \frac{\partial \widehat{\varphi}_{\text {nduct }}}{\partial \psi_{2}} \\
\ldots & \ldots & \ldots & \\
\frac{\partial \widehat{\varphi}_{1}}{\partial \psi_{\text {nduct }}} & \frac{\partial \widehat{\varphi}_{2}}{\partial \psi_{n d u c t}} & \ldots & \frac{\partial \widehat{\varphi}_{\text {nduct }}}{\partial \psi_{\text {nduct }}}
\end{array}\right] \cdot\left[\begin{array}{c}
\psi_{1} \\
\psi_{2} \\
\ldots \\
\psi_{\text {nduct }}
\end{array}\right]+[I d] \cdot\left[\begin{array}{c}
\widehat{\varphi}_{1} \\
\widehat{\varphi}_{2} \\
\ldots \\
\widehat{\varphi}_{\text {nduct }}
\end{array}\right]\right)
\end{gathered}
$$

where by $[I d]$ we mean an identity matrix. The second derivative (Hessian) applying the chain rule, as well, is (see [23]):

$$
\begin{aligned}
\mathbb{D}^{2}(F \circ g) & =\mathbb{D}(\mathbb{D} F \circ g) \mathbb{D} g)= \\
& =(\mathbb{D}(\mathbb{D} F \circ g)) \mathbb{D} g+(\mathbb{D} F \circ g) \mathbb{D}^{2} g= \\
& \left.=\left(\mathbb{D}^{2} F \circ g\right) \mathbb{D} g\right) \mathbb{D} g+(\mathbb{D} F \circ g) \mathbb{D}^{2} g
\end{aligned}
$$

In our case $\mathbb{D}^{2} g=0$. Therefore:

$$
\mathbb{D}^{2}(F \circ g)^{T}=\left[\psi^{\prime}\right]^{T} \cdot\left[\begin{array}{cccc}
\frac{\partial^{2} F}{\partial \psi_{1}^{2}} & \frac{\partial^{2} F}{\partial \psi_{1} \partial \psi_{2}} & \cdots & \frac{\partial^{2} F}{\partial \psi_{1} \partial \psi_{n s e c t}} \\
\frac{\partial^{2} F}{\partial \psi_{2} \partial \psi_{1}} & \frac{\partial^{2} F}{\partial \psi_{2}^{2}} & \cdots & \frac{\partial^{2} F}{\partial \psi_{2} \partial \psi_{n s e c t}} \\
\cdots & \cdots & \cdots & \cdots \\
\frac{\partial^{2} F}{\partial \psi_{n s e c t} \partial \psi_{1}} & \cdots & \cdots & \frac{\partial^{2} F}{\partial \psi_{n s e c t}^{2}}
\end{array}\right] \cdot\left[\psi^{\prime}\right]
$$

Equations (29) and (31) are the general first and second derivatives of the energy dissipation of the network. 
In what follows it is assumed that:

$$
\widehat{\varphi}_{j}=\widehat{\varphi}_{j}\left(\psi_{j}\right)
$$

, i.e., the specific dissipation (pressure loss) in a network section is just a function of the flow through that section $\psi_{j}$. As it will be shown in another article, this is not an obstacle when dealing with branched junctions. This implies that the matrix $\left[\partial \widehat{\varphi}_{j} / \partial \psi_{k}\right]$ in equation 29 is a diagonal matrix, i.e., an entry is zero if $j \neq k$.

Looking at the $i$ component of equation 29) (the stationarity condition of the dissipation) the physical meaning of the previous equations can be clarified. Due to the presence of $\left[\psi^{\prime}\right]^{T}$ we can split the addends into positive and negative. If by $\left\{j, Y\left(x_{i}\right), N\left(x_{n}\right)\right\}$ it is meant that the flow $\psi_{j}$ at duct-section $j$ is influenced by the flow $x_{i}$ but no by $x_{n}$ ( the flow removed from the equations previously), and vice versa, then the terms can be grouped as follows:

$$
\begin{aligned}
\frac{\partial F}{\partial x_{i}}= & \sum_{j, Y\left(x_{i}\right), N\left(x_{n}\right)}\left(\psi_{j} \cdot \frac{\partial \widehat{\varphi}_{j}}{\partial \psi_{j}}+\widehat{\varphi}_{j}\right)-\sum_{j, N\left(x_{i}\right), Y\left(x_{n}\right)}\left(\psi_{j} \cdot \frac{\partial \widehat{\varphi}_{j}}{\partial \psi_{j}}+\widehat{\varphi}_{j}\right)=0 \\
& i \in\{1, \ldots, n-1\}
\end{aligned}
$$

Figure 5 shows graphically the meaning of each component of equations 29) or (33). They are making balances of the terms between brackets over the pseudo-loops which start and end at the same reservoir (the room). Therefore an obvious question arises; when equation 33 , that represents the stationarity condition of the dissipation, is equivalent to the energy balance?, i.e., an equation like (13).

It seems obvious that by assuming the following relationship:

$$
\psi_{j} \cdot \frac{\partial \widehat{\varphi}_{j}}{\partial \psi_{j}}=m \cdot \widehat{\varphi}_{j}
$$

where $m \in \mathbb{R}$ is a constant, we can take out $\widehat{\varphi}_{j}$ as common factor in equation (33) and by dividing by $(m+1)$ we would get an equation like (13). 


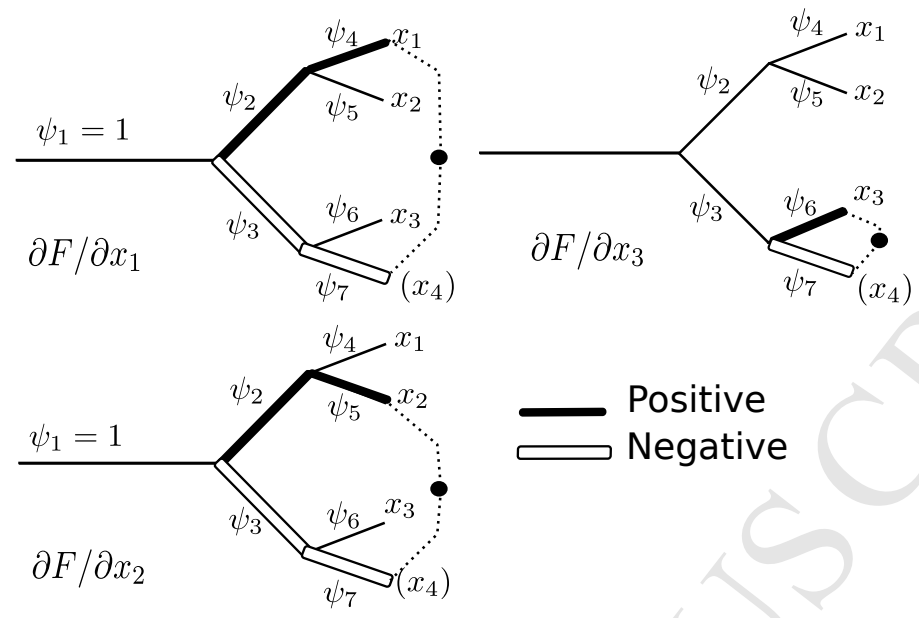

Figure 5: Visualization of the gradient $\nabla F$ for the example network of figure 4

The previous hypothesis 34 leads to:

$$
\begin{aligned}
\frac{d \widehat{\varphi}_{j}}{\widehat{\varphi}_{j}} & =m \cdot \frac{d \psi_{j}}{\psi_{j}} \\
\ln \left(\widehat{\varphi}_{j}\right) & =m \cdot \ln \left(\psi_{j}\right)+\ln \left(\widehat{K}_{j}\right)=\ln \left(\widehat{K}_{j} \cdot \psi^{m}\right) \\
\widehat{\varphi}_{j} & =\widehat{K}_{j} \cdot \psi_{j}^{m}
\end{aligned}
$$

Taking into account the sense of the flows and the equation $\sqrt{35})^{2}$, the dissipation function 16 can be rewritten as:

$$
F\left(\psi_{1}, \ldots, \psi_{n \text { sect }}\right)=\widehat{\varphi}_{T}=\sum_{j=1}^{n s e c t} \widehat{K}_{j} \cdot\left|\psi_{j}\right|^{m-1} \cdot \psi_{j} \cdot \psi_{j}=\sum_{j=1}^{n s e c t} \widehat{K}_{j} \cdot\left|\psi_{j}\right|^{m+1}>0
$$

The dissipation power-law ${ }^{3}$ in equation 35 was, to our knowledge, first discovered by Niven [10]. Note that $\widehat{K}_{j}$ is a positive constant, related to the head loss coefficient so we call it simply, loss coefficient. By substituting equation

\footnotetext{
${ }^{2}$ to take into account, implicitly, the sense of the flow, it is written as $\widehat{\varphi}_{j}=\widehat{K}_{j} \cdot\left|\psi_{j}\right|^{m-1} \cdot \psi_{j}$.

${ }^{3}$ For a horizontal duct/pipe equation 35 is more easily recognized as $\Delta p_{j}=\widehat{K}_{j} \cdot \dot{V}_{j}^{m}$, as appeared in Niven [10.
} 
(35) into equation 33, we can check that the stationarity (extremum) of the network dissipation implies the energy conservation of the conventional closedform solution:

$$
\begin{aligned}
& \sum_{j, Y\left(x_{i}\right), N\left(x_{n}\right)}\left(\psi_{j} m \cdot K_{j} \cdot \psi_{j}^{m-1}+\widehat{\varphi}_{j}\right)-\sum_{j, N\left(x_{i}\right), Y\left(x_{n}\right)}\left(\psi_{j} m \cdot K_{j} \cdot \psi_{j}^{m-1}+\widehat{\varphi}_{j}\right)=0, \\
& \sum_{j, Y\left(x_{i}\right), N\left(x_{n}\right)}\left(m \cdot K_{j} \cdot \psi_{j}^{m}+\widehat{\varphi}_{j}\right)-\sum_{j, N\left(x_{i}\right), Y\left(x_{n}\right)}\left(m \cdot K_{j} \cdot \psi_{j}^{m}+\widehat{\varphi}_{j}\right)=0, \\
& (m+1) \cdot\left(\sum_{j, Y\left(x_{i}\right), N\left(x_{n}\right)} \widehat{\varphi}_{j}-\sum_{j, N\left(x_{i}\right), Y\left(x_{n}\right)} \widehat{\varphi}_{j}\right)=0 \\
& i \in\{1, \ldots, n-1\}
\end{aligned}
$$

Equation (37) is just equal to equation (13) written for the pseudo-loop $i$. Therefore, if we assume that the equation (34) is true and since the energy conservation must be fulfilled at the steady-state solution at every loop, then the dissipation function 21 has a stationary point at same point $\vec{x}^{*}$ as the traditional solution using the closed-form solution of flow network analysis.

Moreover, in order to see that the stationary point of $F$ is a minimum, the second derivative (Hessian, see equation (31)) of $F$ must lead to a definite positive bilinear form. The matrix $\left[\partial^{2} F / \partial \psi_{j} \partial \psi_{k}\right]$ in equation 31 is a diagonal matrix. Any diagonal element has the form:

$$
\frac{\partial^{2} F}{\partial \psi_{j}^{2}}=\frac{\partial^{2} \widehat{\varphi}_{j}}{\partial \psi_{j}^{2}} \cdot \psi_{j}+2 \cdot \frac{\partial \widehat{\varphi}_{j}}{\partial \psi_{j}}
$$


If equation 38 is positive, we can build a matrix like:

$$
\begin{aligned}
& {\left[\sqrt{\mathbb{D}^{2} F}\right]=} \\
& =\left[\begin{array}{cccc}
\sqrt{\frac{\partial^{2} \widehat{\varphi}_{1}}{\partial \psi_{1}^{2}} \cdot \psi_{1}+2 \cdot \frac{\partial \widehat{\varphi}_{1}}{\partial \psi_{1}}} & 0 & \cdots & 0 \\
0 & \sqrt{\frac{\partial^{2} \widehat{\varphi}_{2}}{\partial \psi_{2}^{2}} \cdot \psi_{2}+2 \cdot \frac{\partial \widehat{\varphi}_{2}}{\partial \psi_{2}}} & \cdots & 0 \\
\cdots & \cdots & \cdots & \cdots \\
0 & 0 & \cdots & \sqrt{\frac{\partial^{2} \widehat{\varphi}_{\text {nduct }}}{\partial \psi_{\text {nduct }}^{2}} \cdot \psi_{\text {nduct }}+2 \cdot \frac{\partial \widehat{\varphi}_{\text {nduct }}}{\partial \psi_{\text {nduct }}}}
\end{array}\right]
\end{aligned}
$$

and then the equation (31) can be expressed as:

$$
\begin{aligned}
& \mathbb{D}^{2}(F \circ g)= \\
& {\left[\psi^{\prime}\right]^{T} \cdot\left[\sqrt{\mathbb{D}^{2} F}\right] \cdot\left[\sqrt{\mathbb{D}^{2} F}\right] \cdot\left[\psi^{\prime}\right]=} \\
& {[R]^{T} \cdot[R]}
\end{aligned}
$$

where $[R]$ is a rectangular matrix. There is a well known theorem which says : "A matrix $A$ is positive definite if and only if it can be written as $A=R^{T} \cdot R$ for some possibly rectangular matrix $R$ with independent columns." It is easy to prove since: $x^{T} \cdot A \cdot x=x^{T} R^{T} R x=(R x)^{T} \cdot(R x)=\|R x\|^{2}>0$. Besides, since the columns are independent, $R x \neq 0$ if $x \neq 0$.

Let us see that equation (40) complies this theorem and therefore the stationary point is a minimum. On one hand, the columns of $\left[\psi^{\prime}\right]$ are independent since they represent independent loops of the tree-shaped network. On the other, the condition on equation (38) taking into account equation 35 , leads after some algebra (see annex $\mathrm{A}$ to the equation:

$$
\frac{\partial^{2} F}{\partial \psi_{j}^{2}}=m(m+1) \cdot \widehat{K}_{j} \cdot\left|\psi_{j}\right|^{m-1}>0
$$

Equation 411) is fulfilled whenever the exponent $m \notin[-1,0]$. This result was already obtained by Niven [10] but only for parallel pipes.

In summary, to achieve that the stationary point $\vec{x}^{*}$ of the dissipation 21) was equivalent to the energy conservation in the network, the following is needed: 
- Power-law for the dissipation (equation (35)) at each $j$ section.

- $\widehat{K}_{j}$ the loss coefficient of the $j$ section must be positive.

- The exponent $m \in\{\mathbb{R}-[-1,0]\}$, although it is indeterminate, it must be, -in general-, the same for all the network.

These are the outcomes of a pure mathematical reasoning. Some questions arise: what should the actual value of the exponent $m$ be?, what is the underlying physical meaning of $\widehat{K}_{j}$, moreover are they really constant?, if not, what does it mean?. Next 4 and its subsections try to answer these questions. The discussion is based on the energy dissipation by the simplest and well-studied network element: the straight pipe/duct.

\section{The loss as a power-law in networks.}

In the previous $\$ 3$ it was concluded that the dissipation law, i.e., the conversion of mechanical energy into internal energy as a function of the flow rate must follow a power-law (see equation 35 ). The loss coefficients $\widehat{K}$ must be positive real numbers. The well-known book from Idelchik 24] gathers the head loss coefficients of many fittings and branched junctions. In particular, according to Idelchik, for the case of branched junctions these values can be negative (mainly in the converging cases -return networks-). However, in this case the correct name should be head change not head loss. In [12] B. Schmandt and H. Herwig using CFD made a clear discussion about why these coefficients might attain a negative value. Roughly, the reason relies in the fact that by its traditional definition they include a diffusive exchange of mechanical energy between the junction branches which might overcome the dissipative effect in a branch. Notice, nevertheless, that the branched junction as a whole dissipates mechanical energy. The discussion about how to incorporate these junctions in the MinEP method to estimate the steady-state flow distribution deserves a separated paper and will be delayed. A hint about the solution relies in the redefinition of the so called loss coefficients for junctions. Schmandt's paper 
shows that branched junctions are also dissipative structures, in which a proper loss coefficient $K_{\varphi}>0$ can be defined.

In short, for branched junctions (we insist, mainly in converging ones) it is possible for the pressure drop in a branch to be positive or negative (see 24] and for an explanation [12] ). However, the branched junction, taken as a whole, is a sub-system whose energy dissipation is always positive.

Therefore here, we will focus on systems made up of conduit elements with two-ports and the dissipation at the branched elements will be neglected. This will serve to expose the main fundamental ideas.

First, we make a review of recent advances about the losses occurring in straight pipes (smooth and rough). This will allow us to link the $\widehat{K}_{j}$ and the exponent $m$ with the physics.

We will show how to apply the theory to a network system made up of smooth pipes. In this case the outcomes from $\S 3$ can be applied in a straightforward way. However for networks composed by rough pipes the solution needs a fixed-point iteration since the $\widehat{K}_{j}$ cannot be kept constant . Finally a discussion follows about the chosen value of the exponent $m$ and about how to incorporate the fittings or minor losses.

\subsection{Straight pipe/ducts network systems}

Let us assume that the only dissipative elements are straight ducts (or pipes). The Darcy friction factor $f_{D}$, in pipes has been extensively studied. Although, unfortunately, it still remains more to be done to fully understand the turbulence phenomenon [25].

The $f_{D}$ factor is commonly defined by:

$$
\Delta p=f_{D} \cdot \frac{L}{D_{h}} \cdot \frac{\rho \bar{v}^{2}}{2}
$$

This friction factor is traditionally represented in what is known as Moody's diagram [26. Mc Govern [27] recently published a report with the most recent advances about this Moody's diagram (see figure (6p). In concrete, he prepared variants that include not only monotonic roughness curves, but also inflectional 
roughness curves. The roughness $\epsilon$ (named $k_{s}$ in the specialised literature) that appears in this chart is the equivalent Nikuradse's sand roughness [28] (see [18] [29] 30]). Nikuradse made experiments with pipes coated inside with sand of a certain regular grain-size $\epsilon$ (in other words, densely packed spheres of diameter $\epsilon)$. The equivalence between Nikuradse's roughness and the actual pipe is done experimentally at the complete turbulent zone.

In 2008, 18 Herwig et al. made an insightful paper. Here we summarized some of their findings which are important to understand what follows. They showed that equation 42 is valid for horizontal fully developed flows and that it can be generalised for the dissipation $\widehat{\varphi}$ as:

$$
\widehat{\varphi}=f_{D} \cdot \frac{L}{D_{h}} \cdot \frac{\rho \bar{v}^{2}}{2}
$$

The friction factor in the turbulent region has three different behaviours; the smooth pipe curve, the transition region and the fully/complete turbulent zone. This different behaviour is related to the physical phenomena occurring at the boundary layer.

A smooth pipe is supposed to have no roughness $\epsilon=0$ and the friction factor always decreases with the Reynolds number Re. It can be argued that the friction is controlled by a viscous sub-layer close to the pipe wall.

On the contrary at the transition region the behaviour is very different according to the type of roughness. The original Moody's chart was done for commercial pipes whose roughness is completely random or irregular. This corresponds to the monotonic curves at the transition zone. Therefore the friction factor is a monotonically decreasing curve that departs from the smooth-pipe curve at some point and reaches a value independent of the Reynolds number in the complete turbulent zone. However if the roughness has a regular pattern (Herwig studied T, Q and S-types) then the new inflectional curves appear. In other words, $f_{D}$ departs from the smooth-pipe curve but its behaviour is not monotonical at the transition region. An extreme case is that of Nikuradse experiments with the sand coated pipes (not shown in figure (6)). Nikuradse did 


\section{Darcy Friction Factor for Pipe Flow}

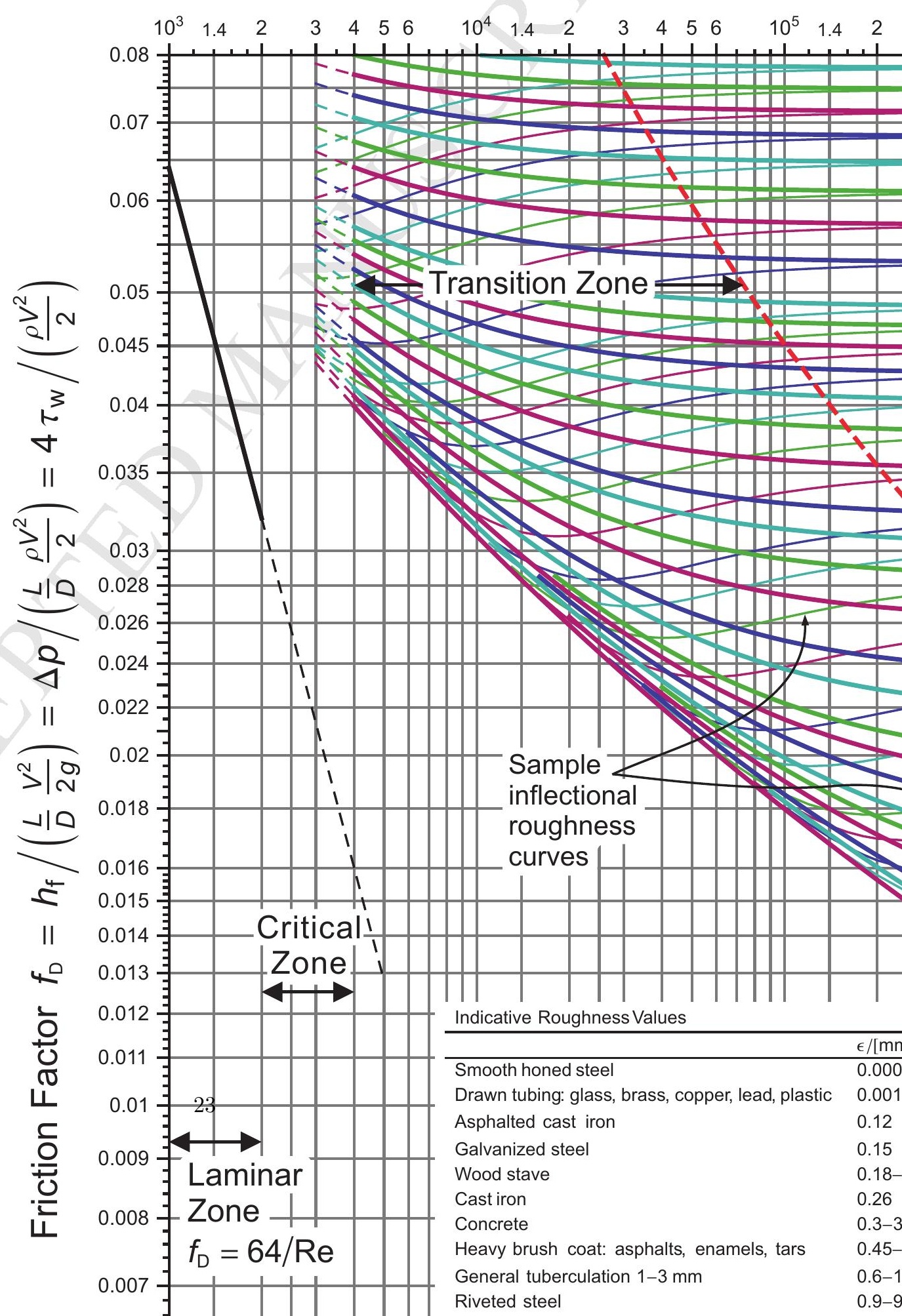


not found a smooth transition zone, but a jump from the smooth-pipe curve to the fully turbulent region.

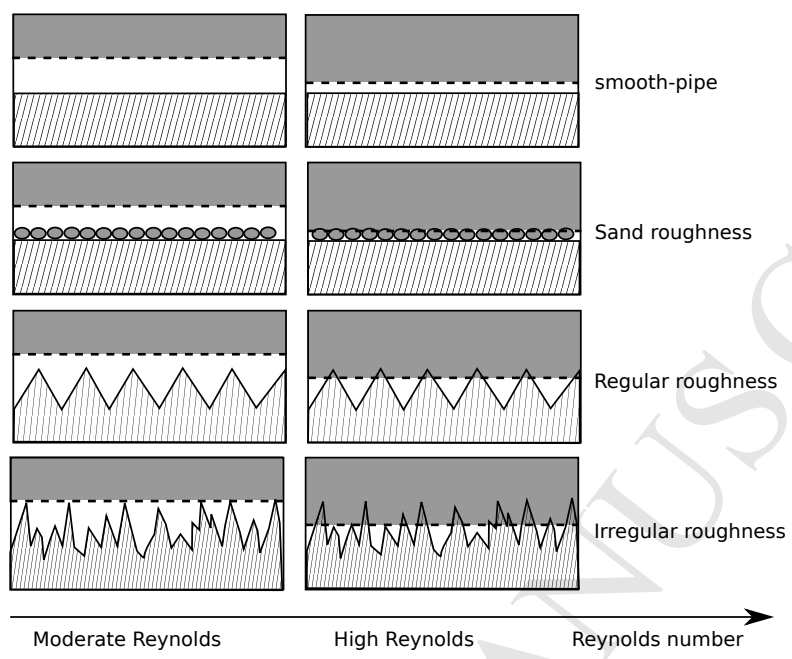

Figure 7: (Adapted from [18):Decreasing height of the viscous sub-layer with increasing Reynolds numbers and the intrusion of sand, regular and irregular roughnesses into it (dashed line: edge of the viscous sublayer)

Figure (7) is a modified version of a figure appearing in [18. Although the full discussion of the wall roughness effects is hard and out of the scope of the paper, that figure serves to grasp the main current ideas which try to explain the behaviour of $f_{D}$ (for more details see for instance [31]). Figure shows that the core of the flow is not aware of the roughness until a high enough Reynolds number is reached. In the case when the roughness is regular (according to Jimenez [31]), it seems that there are two contraposing effects; it creates an extra form or pressure drag, which increases the skin-friction and weakens the viscous generation cycle which decreases it. At high Reynolds the form drag dominates. This creates the inflectional-type curves at the transition. Jimenez also points out that already Colebrook in 1939 suggested that for irregular roughness these effects are built up gradually since "each roughness element" becomes active individually at some Reynolds. This would give a monotonically curve in the transition zone. However, for a completely smooth-pipe the physical 
mechanism for energy dissipation does not change with the Reynolds.

Summarizing for rough pipes, it could be said that from the point of view of the flow, the pipe is not always the same object. At moderate Reynolds the flow "sees" a smooth-pipe but at some Reynolds it becomes aware of the actual geometrical roughness either suddenly or gradually, and thus it is as if the pipe-object was changed.

Therefore it seems that there is a roughness scale which changes with the Reynolds number. In 2007 Noor Afzal [32] proposed an interesting idea. He defined a roughness scale $\phi$, which after using the parameters appearing also in [32, can be rewritten as:

$$
\phi=1+0.306 \cdot \frac{\epsilon}{D} \cdot R e_{D} \cdot \sqrt{\frac{f_{D}}{8}} \cdot \exp \left[-\left(\tilde{j} \frac{1}{(\epsilon / D) \cdot R e_{D}} \sqrt{\frac{8}{f_{D}}}\right)\right]
$$

where $\tilde{j}$ is a free parameter. For $\tilde{j}=0$ the monotonical transition zone is obtained while for $\tilde{j} \neq 0$ an inflectional zone is modelled. Afzal suggests, based on experimental data to use $\tilde{j}=11$. Note also, that the smooth-pipe case $(\epsilon=0)$ is equivalent to $\phi=1$.

Moreover Afzal defines the roughness Reynolds number $R e_{\phi}$ as:

$$
R e_{\phi}=\frac{R e_{D}}{\phi}
$$

This allows him to propose, in his own words, a universal relation (equation (25b) in [32]):

$$
\frac{1}{\sqrt{f_{D}}}=2 \cdot \log _{10}\left(R e_{\phi} \cdot \sqrt{f_{D}}\right)-0.8
$$

When $\phi$ (eq. 44) is used in eq. 446) the following equation is obtained:

$$
\frac{1}{\sqrt{f_{D}}}=-2 \cdot \log _{10}\left[\frac{2.51}{R e_{D} \sqrt{f_{D}}}+\frac{1}{3.7(D / \epsilon)} \cdot \exp \left(\frac{-\tilde{j} \cdot 2.83}{R e_{D} \sqrt{f_{D}}(\epsilon / D)}\right)\right]
$$

Precisely equation (47) was used by Mc Govern in [27] to obtain the diagram of figure $(6)$. 
The reason why Afzal calls equation (46) a universal relation is because figure (6) is transformed just into the curve shown in figure (8).

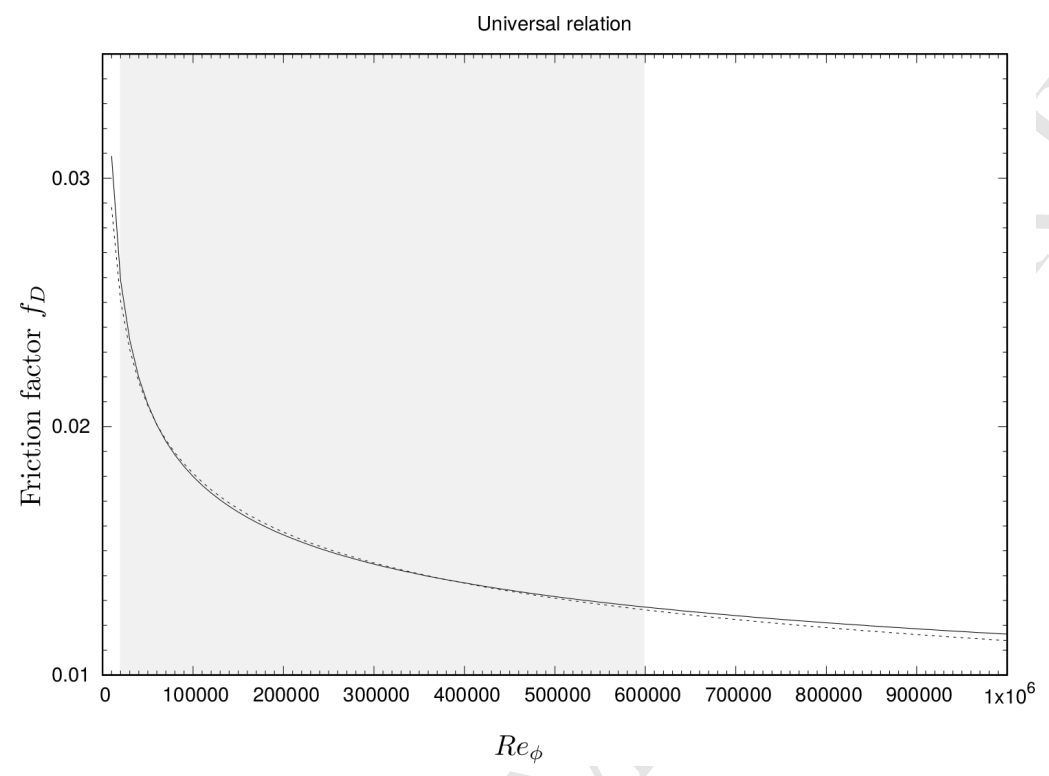

Figure 8: Universal relation (equation 46 solid-line) and a power-law fit 48 (dotted line) using $R e_{\phi} \in[15000,600000]$.

Observe that the $x$-axis is $R e_{\phi}$ and that for a smooth-pipe $R e_{\phi}=R e_{D}$. Equation (46) can be fit very accurately to a power-law as:

$$
f_{D}=K_{f i t} \cdot \operatorname{Re}_{\phi}^{m_{f i t}}=K_{f i t} \cdot\left(\frac{R e_{D}}{\phi}\right)^{m_{f i t}}=\left(\frac{K_{f i t}}{\phi^{m_{f i t}}}\right) \cdot R e_{D}^{m_{f i t}}
$$

For air duct systems in HVAC $R e_{D} \in[15000,600000]$. Making the regression analysis to fit the power-law (48) inside that range, the following values are obtained: $K_{f i t}=0.1847979768, m_{f i t}=-0.2017240066(R-$ squared $=0.998$, standard error $S_{\text {err }}=0,00735475$ and the maximum and minimum relative errors are $0.82 \%$ and $-3.18 \%$ respectively for that $R e_{D}$ range). The range is highlighted in figure (8) and shows the very good fit. The values change slightly with the range of $R e_{D}$ taken. For instance, for a big range like $R e_{D} \in\left[15000,10^{7}\right]$ the regression results are; $K_{f i t}=0.1326376858, m_{f i t}=-0.1751199428(R-$ squared $=0.994$, standard error $S_{\text {err }}=0.0184$ and maximum and minimum 
relative errors are $1.38 \%$ and $-9.56 \%$.

Therefore equation 43 assuming $\bar{v} \cdot(\pi D) / 4=\dot{V}$, can be written as:

$$
\widehat{\varphi}=\frac{K_{f i t}}{\phi^{m_{f i t}}} \cdot\left(\frac{4}{\nu \cdot \pi \cdot D}\right)^{m_{f i t}} \cdot\left(\frac{8 \rho L}{\pi^{2} \cdot D^{5}}\right) \cdot|\dot{V}|^{\left(1+m_{f i t}\right)} \cdot \dot{V}
$$

or in non-dimensional form as:

$$
\widehat{\varphi}=\frac{K_{f i t}}{\phi^{m_{f i t}}} \cdot\left(\frac{4}{\nu \cdot \pi \cdot D}\right)^{m_{f i t}} \cdot\left(\frac{8 \rho L}{\pi^{2} \cdot D^{5}}\right) \cdot \dot{V}_{T}^{\left(2+m_{f i t}\right)} \cdot|\psi|^{\left(1+m_{f i t}\right)} \cdot \psi
$$

In other words, for horizontal smooth-pipes using $m_{f i t}=-0.2017240066$, equation (50) means that the pressure drop $\Delta p=\widehat{\varphi}$ is proportional to the volume flow rate to the $m=1.798$ ( while the Blassius correlation would give $m=1.75)$. In 33 Cory suggests an exponent value in the range between 1.7 and 1.9 in the transition flow and 2 in the fully turbulent zone.

Finally from equation (50) the loss coefficient of a straight duct/pipe has the form:

$$
\widehat{K}=\frac{K_{f i t}}{\phi^{m_{f i t}}} \cdot\left(\frac{4}{\nu \cdot \pi \cdot D}\right)^{m_{f i t}} \cdot\left(\frac{8 \rho L}{\pi^{2} \cdot D^{5}}\right) \cdot \dot{V}_{T}^{\left(2+m_{f i t}\right)}
$$

This equation allow us to link $\widehat{K}$ to the physics for the case of straight pipes/ducts.

\subsection{Straight smooth-pipe network system}

Let us assume that our network is made up of straight smooth pipes/ducts, without any fittings like elbows, reductions and as aforementioned, the effect of branched junctions is negligible. In order to simplify things, in each section there is only one pipe of a certain size.

According to $\$ 3$ the algorithms 1 and 2 are enough to solve for the steadystate flow distribution through the network in just one minimisation step. Note that in this case for any $j$-section, the roughness scale $\phi_{j}=1$ and therefore the loss coefficient $\widehat{K}_{j}$ (eq. 51) does not depend on the section flow rate $\psi_{j}$. In other words, for a given geometry, the $\widehat{K}_{j}$ is constant. 


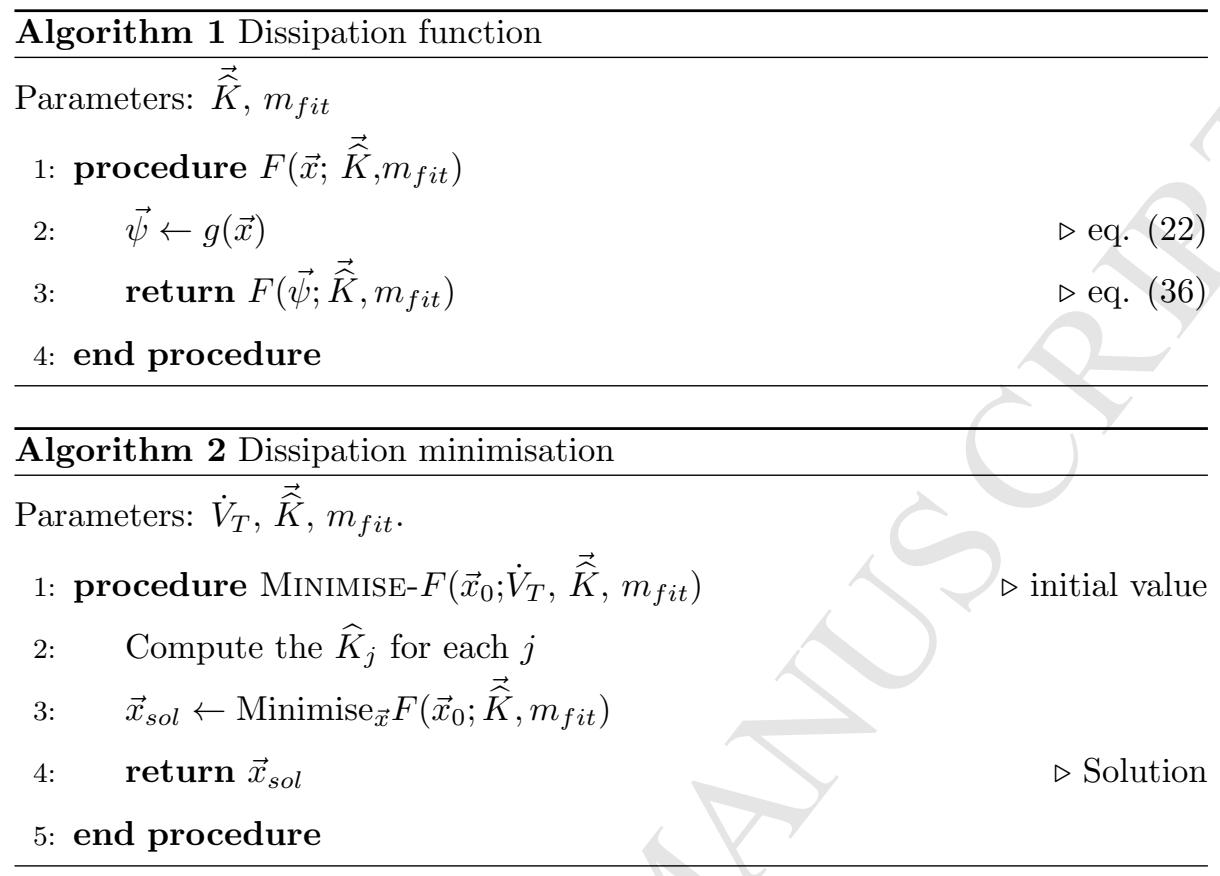

\subsection{Straight rough pipe network system}

According to the discussion in $\S \$ 4.1$ for rough pipes the roughness scale $\phi \neq 1$. This means that the loss coefficients $\widehat{K}$ are, additionally, a function of the flow rate. Note that the dissipation function (36) contains all the physics inside the loss coefficient vector $\overrightarrow{\widehat{K}}$. Therefore the function is not explicitly aware about the physical mechanisms by which a certain $\overrightarrow{\widehat{K}}$ is obtained. In some sense each $\widehat{K}$ is like a black-box. Some variables like pipe length $L$ or the diameter $D$ can be used as "external" controls to modify the $\widehat{K}$ value. However, the roughness scale $\phi$ seems to act as an "internal" control - not directly accessible - which modifies the actual pipe that the flow "sees". Moreover, it can modify the balance among the physical dissipation mechanisms as in the case of the inflectional transition zone. As shown in 4.1, this does not happen in smooth pipes and a certain $m_{f i t}$ (and $K_{f i t}$ ) can be found, that renders $\widehat{K}$ independent of the flow rate.

Therefore for rough pipes we need to search for the correct $\widehat{K}$ that is "compatible", "coherent" or that "matches" the roughness scale $\phi$ imposed by the 
obtained flow rate from the minimisation step.

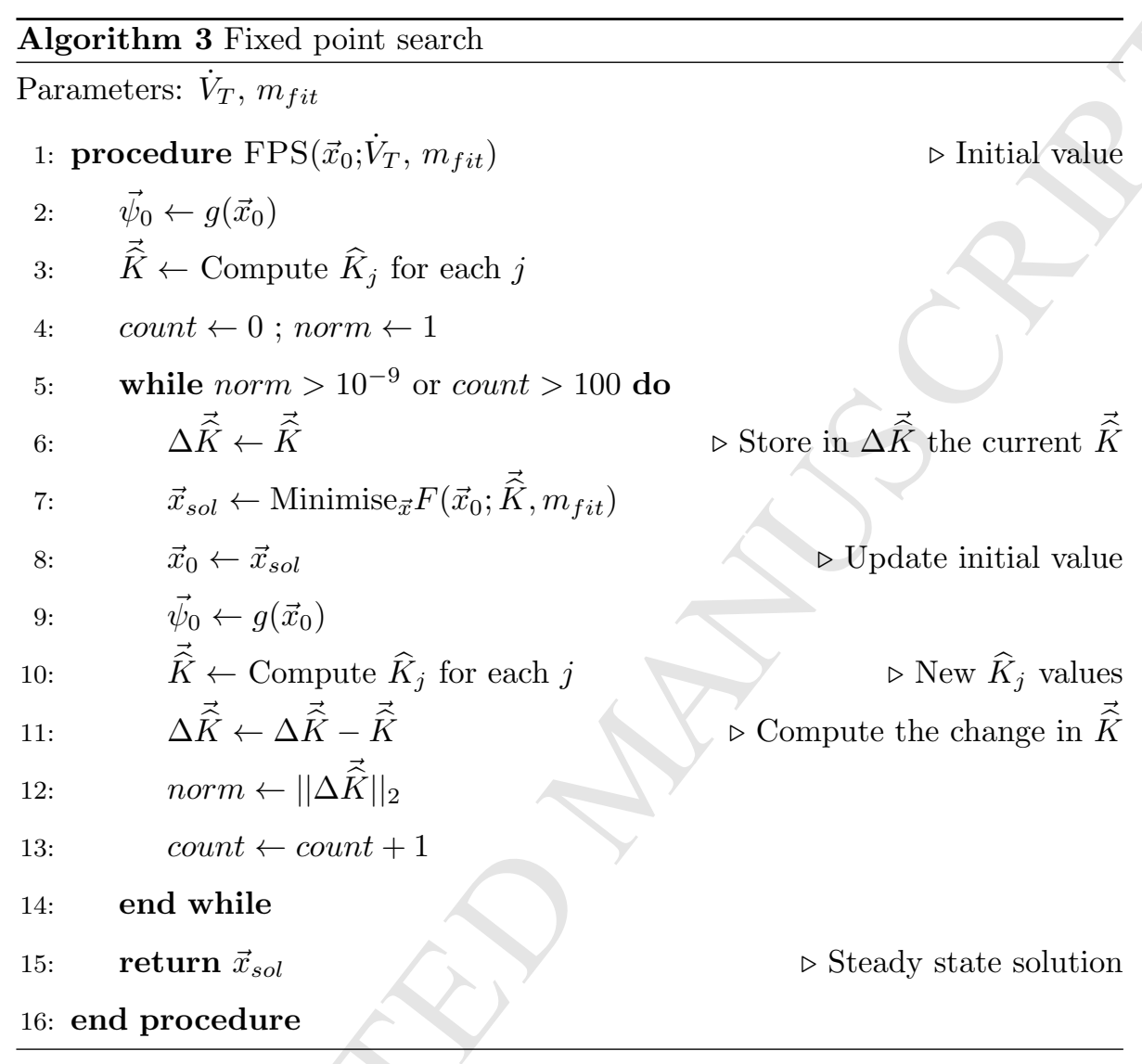

Algorithm (3) is a simple implementation of such search. It uses the two previous algorithms. The minimisation step 2 is inside the while loop. The difference is that now $\widehat{K}_{j}$ depends on the roughness scale $\phi_{j}$ which in turn depends on the flow rate $\psi_{j}$ obtained at section $j$. There is an implicit dependency in $\phi$ (see equations 44 and (47)). Therefore the roughness scale is cleared from equation (48) after solving for $f_{D}$, as:

$$
\phi=\left(\frac{K_{f i t}}{f_{D}}\right)^{1 / m_{f i t}} \cdot R e_{D}
$$

and used for the computation of $\widehat{K}_{j}$ using equation 51 . Note that the $\phi$ in equation 52 depends on $\left\{R e_{D}, \epsilon, D, \tilde{j}\right\}$. 


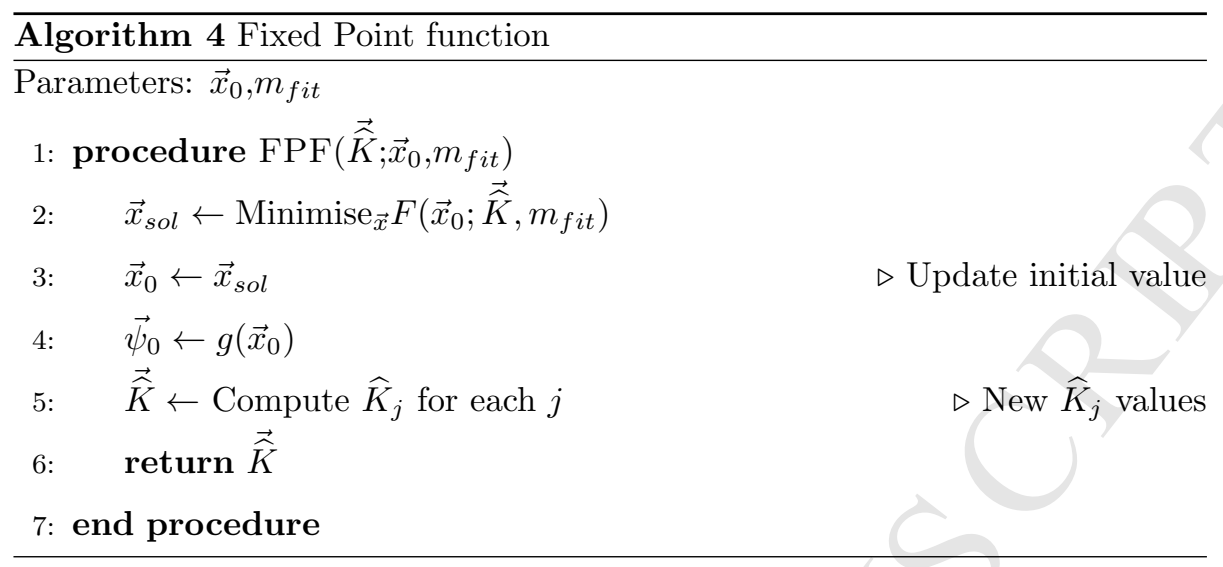

In fact, Algorithm (3) can be viewed as a fixed point function search, since it is a map from $\overrightarrow{\widehat{K}} \in \mathbb{R}_{+}^{n s e c t}$ (i.e. with positive coordinates) to itself. The fixed point function could be defined as $F P F(\overrightarrow{\widehat{K}})$ in Algorithm (4). Once the correct fixed point $F P F\left(\vec{K}^{*}\right)=\vec{K}^{*}$ is obtained, that is, the one that is compatible with the flows obtained at the minimisation step, then this is the correct distribution of the flow through the network. Therefore finding one implies finding the other.

4.4. About the role of the exponent $m$ of the power-law (eq. (35))

As mentioned in $\$ 3$ the exponent $m$ must be the same for the whole network. In $\S 4.1$ it was shown that the Afzal's universal relation allows to chose a certain $m_{f i t}$ for straight pipes. However the pressure loss in fittings (elbows, reductions, etc.) normally is given as a function of $\bar{v}^{2}$.

The selected $m$ does not make a real difference. In other words, once an exponent $m$ is chosen for all the elements of the network, it is possible to apply the Algorithm (3) and the solution will be the same regardless of the $m$ value. Obviously, there are differences. Changing $m$, requires a reformulation of the loss coefficients and the number of iterations to get to the solution, might be different.

Let us assume that Afzal's universal relation is not used. This is equivalent to set $m_{f i t}=0$ and $K_{f i t}=f_{D}$, that is, the specific dissipation (pressure loss) now is proportional to the square of the flow rate $\psi$ (i.e., $m=2$ instead of 
$m=1.798)$.

$$
\widehat{\varphi}=f_{D} \cdot\left(\frac{8 \rho L}{\pi^{2} \cdot D^{5}}\right) \cdot \dot{V}_{T}^{2} \cdot|\psi| \cdot \psi
$$

Now the loss coefficient is written as:

$$
\widehat{K}=f_{D} \cdot\left(\frac{8 \rho L}{\pi^{2} \cdot D^{5}}\right) \cdot \dot{V}_{T}^{2}
$$

Equation 54 means that now even for the case of smooth-pipes the $\widehat{K}_{j}$ depends on the flow rate $\psi_{j}$. Therefore for a network composed only of straight smooth-pipes, Algorithm (2) is not enough and Algorithm (3) must be employed instead. It could be said that a pure minimisation problem has been transformed into an equivalent fixed point one. Anyhow the flow distribution at the steadystate will be the same.

\subsection{Fittings}

The dissipation (pressure loss in fittings) has the form:

$$
\widehat{\varphi}=C_{f i t t i n g} \cdot \frac{\rho \bar{v}^{2}}{2}
$$

Using the volume flow rate it can be rewritten as:

$$
\widehat{\varphi}=C_{\text {fitting }} \cdot \frac{8 \rho}{\pi^{2} D^{4}} \cdot \dot{V}_{T}^{2} \cdot|\psi| \psi
$$

This corresponds to the case $m=2$ and the loss coefficient is given in this case by:

$$
\widehat{K}_{f i t t i n g, 2}=C_{f i t t i n g} \cdot \frac{8 \rho}{\pi^{2} D^{4}} \cdot \dot{V}_{T}^{2}
$$

If other exponent is chosen, by using, for instance, the fit to the Afzal's 
relation (i.e., using $m_{f i t}$ ) then the expression is:

$$
\begin{aligned}
& \widehat{\varphi}=C_{f i t t i n g} \cdot \frac{8 \rho}{\pi^{2} D^{4}} \cdot \dot{V}_{T}^{2} \cdot \frac{1}{|\psi|^{m_{f i t}}} \cdot|\psi| \cdot \psi \cdot|\psi|^{m_{f i t}} \\
& \widehat{\varphi}=C_{f i t t i n g} \cdot \frac{8 \rho}{\pi^{2} D^{4}} \cdot \dot{V}_{T}^{2} \cdot \frac{1}{|\psi|^{m_{f i t}}} \cdot|\psi|^{\left(1+m_{f i t}\right)} \cdot \psi \\
& \widehat{\varphi}=C_{f i t t i n g} \cdot \frac{8 \rho}{\pi^{2} D^{4}} \cdot \dot{V}_{T}^{\left(2+m_{f i t}\right)} \cdot \frac{1}{|\psi|^{m_{f i t}} \cdot \dot{V}_{T}^{m_{f i t}}} \cdot|\psi|^{\left(1+m_{f i t}\right)} \cdot \psi
\end{aligned}
$$

and the loss coefficient is written as:

$$
\widehat{K}_{f i t t i n g, m_{f i t}}=C_{f i t t i n g} \cdot \frac{8 \rho}{\pi^{2} D^{4}} \cdot \frac{1}{|\psi|^{m_{f i t}} \cdot \dot{V}_{T}^{m_{f i t}}} \cdot \dot{V}_{T}^{\left(2+m_{f i t}\right)}
$$

\section{Examples}

This section is dedicated to illustrate what has been said in the previous sections with some exercises.

The example networks are shown in figure 9 and their parameters can be found in table (1). The three following cases have been solved:

- Network system made up exclusively of straight tubes; smooth and rough.

- Network with fittings using $m_{f i t}$.

- Network with fittings using $m=2$.

The air properties are evaluated at $T_{d b}=20\left[{ }^{\circ} \mathrm{C}\right]$ and humidity ratio $W=$ $0.008\left[\mathrm{~kg}_{\mathrm{H}_{2} \mathrm{O}} / \mathrm{kg}_{d a}\right] ; \rho=1.20657\left[\mathrm{~kg} / \mathrm{m}^{3}\right], \nu=1.49389 \cdot 10^{-5}\left[\mathrm{~m}^{2} / \mathrm{s}\right]$. The mean velocity imposed at section 1 is $\bar{v}_{1}=7\left[\mathrm{~ms}^{-1}\right]$, or according to the diameter at that section $\dot{V}_{T}=1.9792\left[\mathrm{~m}^{3} \mathrm{~s}^{-1}\right]$. For the minimisation (Algorithm (2)) we have used the Nelder-Mead algorithm (with a tolerance in the search space of $10^{-9}$ ). This algorithm does not require to evaluate the derivatives of the objective function. In practice a more specific method could be employed. 


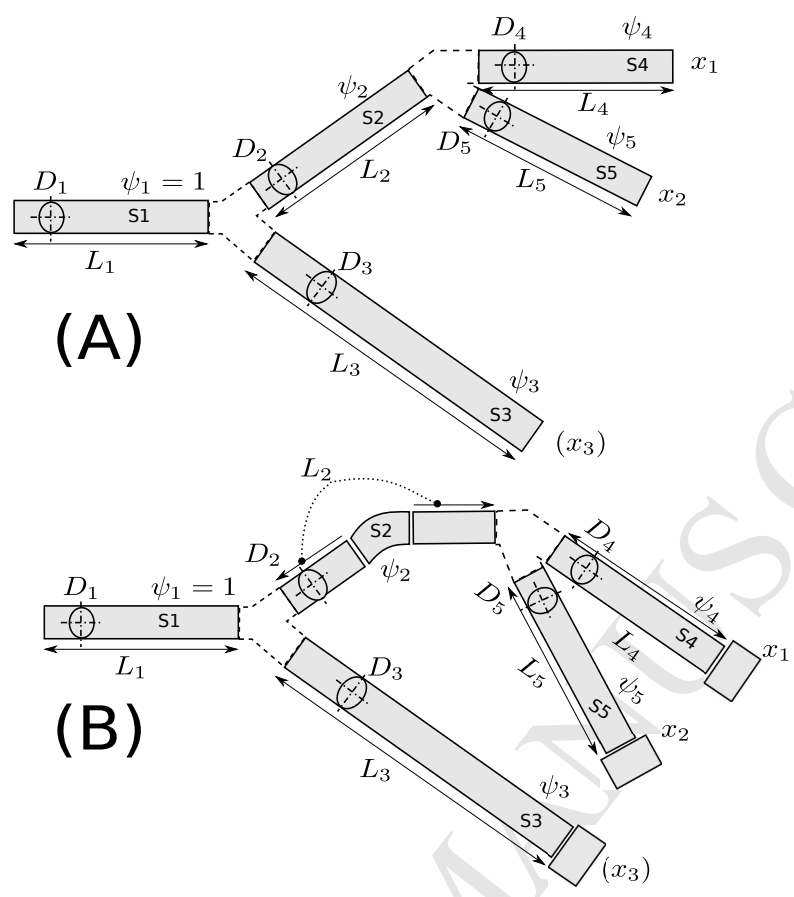

Figure 9: Example networks. (A) without fittings only straight ducts.(B) With fittings, one elbow and diffusers. The values are collected in table (1)

\subsection{Straight ducts network (smooth and rough)}

We start by the simplest case; only straight smooth ducts . First let assume that the tubes are smooth and that the the Afzal's universal relation has been fit between the Reynolds range $[15000,600000]$ (see $\S \$ 4.1$ ). In this case it suffices to use Algorithm (2) and $m_{f i t}=-0.2017240066(m=1.798)$. The initial state value is taken $x_{1}=0.5$ and $x_{2}=0.4$ and the solution is $x_{1}=\psi_{4}=x_{2}=\psi_{5}=$ 0.3549206 . The total pressure loss is $\Delta p_{T}=12.7246[\mathrm{~Pa}]$ and the dissipated mechanical power is $\widehat{\varphi}_{T} \cdot \dot{V}_{T}=25.18[W]$.

If we add roughness to the ducts then we must use Algorithm (3). As was said, the $m$ value chosen for the potential-law for the whole network is an open question. We have used both the one obtained by the fit to the Afzal's universal relation (as was done for the smooth case $m_{f i t}=-0.2017240066(m=1.798)$ ) and also with $m=2$. The solution in both cases is exactly the same ( therefore 


\begin{tabular}{lrrrrr} 
Section & $L[m]$ & $D[m]$ & $\epsilon_{\text {smooth }}$ & $\epsilon_{\text {rough }}$ & $C_{\text {fitting }}$ \\
\hline & & & & & \\
S1 & 10 & 0.6 & 0 & 0.14 & 0.00 \\
S2 & 5 & 0.5 & 0 & 0.14 & 0.22 \\
S3 & 10 & 0.4 & 0 & 0.14 & 1.00 \\
S4 & 1 & 0.4 & 0 & 0.14 & 1.00 \\
S5 & 1 & 0.4 & 0 & 0.14 & 1.00
\end{tabular}

Table 1: Parameters of the example duct network of figure (9). The roughness value corresponds to galvanized steel.

\begin{tabular}{lrrrr} 
Section & $\widehat{K}\left[\mathrm{Jm}^{-3}\right] \equiv[\mathrm{Pa}]$ & $\dot{V}\left[\mathrm{~m}^{3} \mathrm{~s}^{-1}\right]$ & $R e$ & $\bar{v}\left[\mathrm{~ms}^{-1}\right]$ \\
\hline & & & & \\
S1 & 7.2457696 & 1.979200 & 281146 & 7.00 \\
$\mathrm{~S} 2$ & 8.6893641 & 1.404920 & 239483 & 7.15 \\
$\mathrm{~S} 3$ & 50.701286 & 0.574283 & 122365 & 4.57 \\
$\mathrm{~S} 4$ & 5.0701286 & 0.702460 & 149677 & 5.59 \\
$\mathrm{~S} 5$ & 5.0701286 & 0.702460 & 149677 & 5.59
\end{tabular}

Table 2: Example duct network: smooth ducts. figure 9(A)) steady-state solution using $m_{\text {fit }}$. 


\begin{tabular}{lrrrrr} 
Section & $\widehat{K}_{m_{f i t}}\left[\mathrm{Jm}^{-3}\right]$ & $\widehat{K}_{2}\left[\mathrm{Jm}^{-3}\right]$ & $\dot{V}\left[\mathrm{~m}^{3} \mathrm{~s}^{-1}\right]$ & $R e$ & $\bar{v}\left[\mathrm{~ms}^{-1}\right]$ \\
\hline S1 & 85.524209 & 85.524209 & 1.979200 & 281146 & 7.00 \\
S2 & 113.92824 & 121.96710 & 1.411560 & 240614 & 7.20 \\
S3 & 693.42272 & 892.10513 & 0.567644 & 120951 & 4.52 \\
S4 & 72.451495 & 89.203863 & 0.705780 & 150384 & 5.62 \\
S5 & 72.451495 & 89.203863 & 0.705780 & 150384 & 5.62
\end{tabular}

Table 3: Example duct network: rough ducts without fittings. Figure 9) (A) steady-state solution -fixed point- using either $m_{f i t}\left(\widehat{K}_{m_{f i t}}\right)$ or $m=2\left(\widehat{K}_{2}\right)$.

only one set of values is shown). The difference relies on the amount of iterations needed to find the fixed point. The initial value is always the same as in the smooth case. Using $m_{f i t}$ Algorithm (3) takes 7 iterations while using $m=2$ takes 9. The solution is : $x_{1}=x_{2}=0.3565978$. The total pressure loss is $\Delta p_{T}=158.906[\mathrm{~Pa}]$ and the dissipated mechanical power is $\widehat{\varphi}_{T} \cdot \dot{V}_{T}=314.5[\mathrm{~W}]$

\subsection{Network made up of rough straight ducts and fittings}

This case corresponds to figure (9) case $(B)$. In this case Algorithm (3) must also be employed. It has been solved as for the case of rough ducts, for two different values of $m$. In the case of using $m_{f i t}$ the number of iterations was 10 while for $m=2$, it was 5 . The steady-state flows are exactly the same: $x_{1}=x_{2}=0.3489582$. The total pressure loss is $\Delta p_{T}=180.6[\mathrm{~Pa}]$ and the dissipated mechanical power $\widehat{\varphi}_{T} \cdot \dot{V}_{T}=357.4[W]$.

\subsection{Visualization of the fixed point iteration}

Figure (10) serves to illustrate the Algorithm (3). The $z$-axis represents the dissipation (eq. 21) as a function of the independent flow rate vector $\vec{x}$ $(x, y$-plane) for the example network shown in figure $9(\mathrm{~B}))$. The surfaces have been computed by giving values to $x_{1} \in[0,1]$ and $x_{2} \in[0,1]$ and $x_{1}+x_{2} \leq 1$. For a given pair $\left(x_{1}, x_{2}\right)$ the flow rate $\psi_{j}$ at every section is known and then each $\widehat{K}_{j}$ can be computed. If the Reynolds number is below a threshold then 


\begin{tabular}{lrrrrr} 
Section & $\widehat{K}_{m_{\text {fit }}\left[\mathrm{Jm}^{-3}\right]}$ & $\widehat{K}_{2}\left[\mathrm{Jm}^{-3}\right]$ & $\dot{V}\left[\mathrm{~m}^{3} \mathrm{~s}^{-1}\right]$ & $\operatorname{Re}$ & $\bar{v}\left[\mathrm{~ms}^{-1}\right]$ \\
\hline S1 & 85.524209 & 85.524209 & 1.979200 & 281146 & 7.00 \\
S2 & 125.97395 & 135.45323 & 1.381320 & 235459 & 7.04 \\
S3 & 818.25506 & 1041.7404 & 0.597885 & 127394 & 4.76 \\
S4 & 193.15424 & 238.85692 & 0.690659 & 147162 & 5.49 \\
S5 & 193.15424 & 238.85692 & 0.690659 & 147162 & 5.49
\end{tabular}

Table 4: Example duct network: rough ducts with fittings, figure 9) (B) steady-state solution using either $m_{f i t}\left(\widehat{K}_{m_{f i t}}\right)$ or $m=2\left(\widehat{K}_{2}\right)$.

a laminar regime is assumed and the friction factor is computed with the wellknown expression:

$$
f_{D}=\frac{64}{R e_{D}}
$$

Following the interesting discussion of Martyushev [34, we have chosen to used the following relationship for the critical Reynolds $\operatorname{Re}_{D, c}$ for the laminarturbulent transition:

$$
R e_{D, c}=\left(\frac{64}{K_{f i t}}\right)^{\left(1 /\left(m_{f i t}+1\right)\right)}
$$

In our case the fit in the range $R e \in\left[15000,6 \cdot 10^{5}\right]$ leads to $R e_{D, c}=1518$. According to Martyushev, normally the transition is assumed to happen at $R e_{D, c}=2300$, but this depends on the disturbances on the flow. The previous value would represent a lower bound for $R e_{D, c}$.

Finally the dissipation of the network is obtained by using equation 36.

On one hand, if the system consists only of smooth straight ducts and the fit to the Afzal's universal relation is used, then any $\widehat{K}_{j}$ is independent of the flow rate $\psi_{j}$ and $\overrightarrow{\hat{K}}$ would be the same for the whole range of $\vec{x}$. The shape of the dissipation surface for this case is similar to the ones shown in figure 10$]$. In just one minimisation step the steady-state solution is found, so the jumps shown in that figure would correspond to the minimisation process. 
On the other hand, in the general case $\overrightarrow{\widehat{K}}$ varies with $\vec{x}$. That means we must do a fixed point search. Given an initial value for the flows $\vec{x}_{0}$ the corresponding vector of loss coefficients is found $\overrightarrow{\widehat{K}}^{(0)}$ ( see figure 10). After a minimisation step (with a previously chosen power-law , i.e., $m$ ) a new point is reached $\vec{x}_{1}$ and a new vector can be computed $\overrightarrow{\widehat{K}}^{(1)}$, and so on until a fixed point $\overrightarrow{\widehat{K}}^{(*)}$ is reached. In other words, in the general case, each jump in figure 10 represents a minimisation step (see Algorithm (2)).

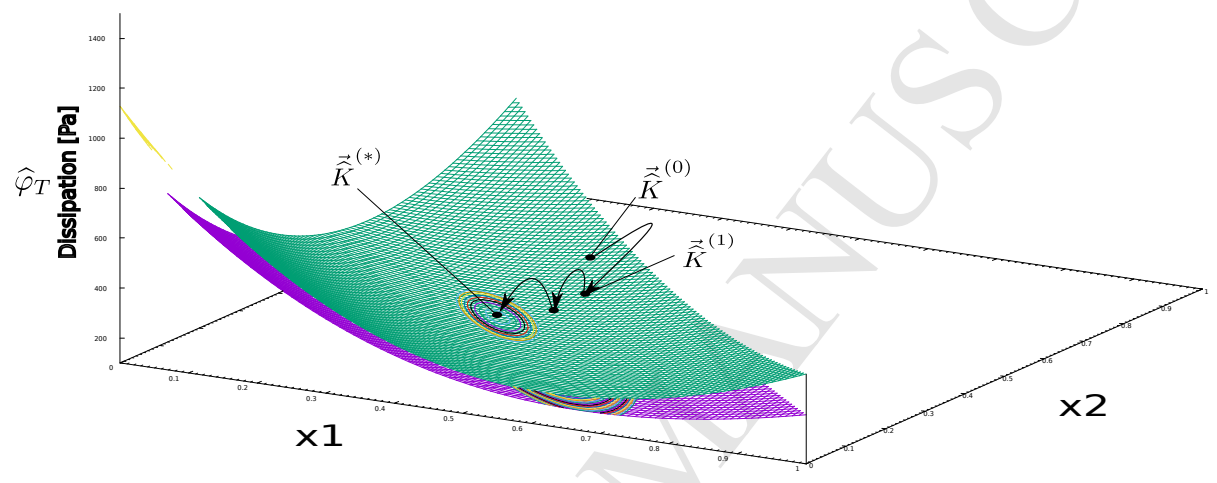

Figure 10: Total pressure loss $\Delta p_{T} \equiv \widehat{\varphi}_{T}$ as a function of the distribution flow rates for the network of figure 9 . Rough ducts. Bottom surface corresponds to the case without fitting (case (A)). Upper surface includes the effect of the fittings (case (B)).

\section{Discussion}

This section gathers the discussion of some aspects that were left out previously to allow a better exposition.

\subsection{Flow sense}

The dissipation function is a positive definite function. It depends on the absolute value of the flow rates $\psi_{j}$ and on the positive loss coefficients $\widehat{K}_{j}$. However, in a practical calculation the flow rates could be negative (reverse their sense). This is taken into account by the value of the $\widehat{K}_{j}$ which might change upon a flow sense reversion. The system under analysis may have symmetrical objects (like straight ducts or pipes or constant cross area elbows), but also 
unsymmetrical ones like a reduction (which would turn into an expansion, thus changing its loss coefficient).

For instance, in our example of a duct network made up of straight tubes, the resulting steady-state flow distribution will be the same regardless of taking the $\dot{V}_{T}$ as a supply or a return flow.

\subsection{Dissipation as a measure}

The results in $\sqrt[3]{3}$ seem to indicate that the dissipation function (eq. (36) is a kind of a measure. It can be considered as an $\ell_{(m+1)}$ norm, since it can be written as:

$$
\left\|\widehat{\varphi}_{T}\right\|_{(m+1)}=\sqrt[(m+1)]{\sum_{j=1}^{n s e c t}\left|\sqrt[(m+1)]{\widehat{K}_{j}} \cdot \psi_{j}\right|^{(m+1)}}
$$

All the physics is enclosed into the loss coefficients $\widehat{K}_{j}$. It seems that if the physical dissipation mechanism, the one that depends on the flow rate, remains the same then it is possible to find a proper $m$ which leaves $\widehat{K}_{j}$ constant. That is the case for instance of smooth-pipes/ducts in the turbulent regime (by using the $\left.m_{f i t}\right)$ or the linear dependence of the dissipation with the flow rate for the laminar regime $(m=1)$. In this cases a single dissipation power-law can be found. In the smooth-pipes system if the correct $m$ is used then the minimisation step of Algorithm (2) is enough. If, on the contrary, the dissipation function is not correctly tuned, then the same problem looks like and turns into a fixed point problem (Algorithm (3)).

Although in the examples above, the number of iterations was shown for completeness, the paper does not analyse the effect of choosing a certain exponent $m$ on the speed of finding the solution.

Anyhow, always in real practice there will be several physical mechanisms playing different roles and it will not be possible to tune the dissipation occurring through the network to a single power-law (see the Cory's comments [33] at this respect). Therefore the problem, in practice, will be always of the fixed point type. 


\subsection{Type of extremum of the fixed point solution}

Finally, although it was shown that if the loss coefficients are constant the stationary point is a minimum, when dealing with the fixed point problem this is not guaranteed. In fact, there could be more than one fixed point, each one with a different nature (i.e. local minimum or maximum). For the cases shown it seems to be globally also a minimum and therefore the idea of using a MinEP principle could still be applied although hidden in a fixed point function problem.

However, this point and its practical implications deserves further analysis in future researches.

\section{Conclusions}

The paper shows under which conditions the MinEP (Minimum Entropy Production) could be equivalent to the standard close-form solutions employed in flow network analysis. The MinEP for isothermal and incompressible flows has been shown to be equivalent to the Minimum Energy Dissipation.

It has been shown that although the same power-law (same exponent $m$ ) must be used for the whole network and the loss coefficient must be constant, these are not real obstacles for its application. In fact, the interplay of the loss coefficients with the flow rate seems to hide a deeper physical content and some understanding about its nature has been pointed out. The combination of the minimisation step with the physical dependence of the $\widehat{K}_{j}$ on the flow rate, leads to a fixed point function formulation of the problem. The solution of this latter problem seems to lead to the stationary point or points of a dissipation function. This new function seems to have also a minimum at the solution but the properties of this function related to the dissipation of the individual components of the network should be studied further.

Although we think this new method has advantages in the case of return networks with branched junctions, these latter have not been included in the current analysis in order to present the main ideas. However, it was pointed 
out that they can be included in the current analysis but it will be discussed in detail in another paper.

Finally, despite our main target has been to find the steady-state flow distribution of a given tree-shaped HVAC duct-network, the extension to other types of networks will be taken as a future work.

\section{Acknowledgements}




\section{Appendix A Second derivative of $F$}

Since:

$$
\widehat{\varphi}=K \cdot|\psi|^{m-1} \cdot \psi
$$

We have:

$$
\begin{aligned}
\frac{\partial \widehat{\varphi}}{\partial \psi} & =K|\psi|^{m-1}+K(m-1) \psi^{2}|\psi|^{m-3} \\
\frac{\partial^{2} \widehat{\varphi}}{\partial \psi^{2}} & =3 K(m-1)|\psi|^{m-3} \psi+K(m-1)(m-3)|\psi|^{m-5} \psi^{3}
\end{aligned}
$$

Therefore according to equation 38 :

$$
\begin{aligned}
& \left.\frac{\partial^{2} F}{\partial \psi^{2}}=2 K|\psi|^{m-1}+5 K(m-1)|\psi|^{(} m-3\right) \psi^{2}+K(m-1)(m-3)|\psi|^{m-5} \psi^{4} \\
& \frac{\partial^{2} F}{\partial \psi^{2}}=|\psi|^{m-5} K\left[2|\psi|^{4}+5(m-1)|\psi|^{2} \psi^{2}+(m-1)(m-3) \psi^{4}\right] \\
& \frac{\partial^{2} F}{\partial \psi^{2}}=|\psi|^{m-5} K[2+5(m-1)+(m-1)(m-3)]|\psi|^{4} \\
& \frac{\partial^{2} F}{\partial \psi^{2}}=K m(m+1)|\psi|^{m-1}
\end{aligned}
$$

\section{References}

[1] P. A. Domanski, H. I. Henderson, W. V. Payne, Sensitivity analysis of installation faults on heat pump performance, Tech. rep., National Institute of Standards and Technology Technical, DOI 10.6028/NIST.TN.1848 (2014).

[2] A. Bejan, Entropy generation minimization : the method of thermodynamic optimization of finite-size systems and finite-time processes, CRC Press, Boca Raton, 1996.

[3] A. Bejan, G. Tsatsaronis, M. Moran, Thermal design and optimization, John Whiley \& Sons, 1996, ISBN: 0-471-58467-3. 
[4] H. Herwig, The role of entropy generation in momentum and heat transfer, Journal of heat transfer 134. doi:10.1115/1.4005128].

[5] T. Wenterodt, C. Redecker, H. Herwig, Second law analysis for sustainable heat and energy transfer: The entropic potential concept, Applied Energy 139 (2015) 376-383, dOI 10.1016/j.apenergy.2014.10.073.

[6] H. Herwig, B. Schmandt, How to determine losses in a flow field: A paradigm shift towards the second law analysis, Entropy 16 (6) (2014) 2959-2989. doi:10.3390/e16062959

URL http://www.mdpi.com/1099-4300/16/6/2959

[7] H.-C. Zhang, Y. Guo, H. Yu, L. Yan, Y. Ji, Y. Li, Estimation of loss coefficient for conduit bends with circular cross section by the second law approach, Heat Transfer Research 47 (2016) 203-217.

[8] A. Bejan, S. Lorente, The constructal law and the thermodynamics of flow systems with configuration, International Journal of Heat and Mass Transfer 47 (14) (2004) 3203 - 3214. doi:https://doi.org/10.1016/j.ijheatmasstransfer.2004.02.007.

URL http://www.sciencedirect.com/science/article/pii/ S0017931004000523

[9] E. Sciubba, Entropy generation minima in different configurations of the branching of a fluid-carrying pipe in laminar isothermal flow, Entropy 12 1855-1866, dOI 10.3390/e12081855.

URL http://www .mdpi . com/1099-4300/12/8/1855

[10] R. Niven, Simultaneous extrema in the entropy production for steady-state fluid flow in parallel pipes, Arxivdoi::10.1515/jnetdy.2010.022.

URL https://arxiv.org/abs/0911.5014v2

[11] R. Niven, Steady state of a dissipative flow-controlled system and the maximum entropy production principleArXiv:0902.1568 [physics.class-ph]. doi:DOI10.1103/PhysRevE.80.021113. 
[12] B. Schmandt, H. Herwig, The head change coefficient for branched flows: Why losses due to junctions can be negative, International Journal of Heat and Fluid Flow 54 (2015) 268 - 275. doi:https://doi.org/10.1016/j.ijheatfluidflow.2015.06.004 URL http://www.sciencedirect.com/science/article/pii/ S0142727X15000697

[13] H. Herwig, The misleading use of enthalpy in an energy conversion analysis, Natural Science 6 878-885. doi:10.4236/ns.2014.611085.

[14] F. Durst, Fluid Mechanics. An introduction to the theory of fluid flows, Springer-Verlag, 2008, ISBN:978-3-540-71342-5.

[15] H. Herwig, Strömungsmechanik, Vieweg+Teubner Verlag, 2008, ISBN: 9783-8348-0334-4.

[16] H. Herwig, B. Schmandt, Loss coefficients for compressible flows in conduit components under different thermal boundary conditions, Proceedings of the 15th International Heat Transfer Conference, IHTC-15, Kyoto (Japan) 15 (2014) 2071-2084, paper 8482.

[17] R. P. Benedict, Fundamentals of pipe flow, John Wiley \& Sons, 1980, ISBN 0-471-03375-8.

[18] H. Herwig, D. Gloss, T. Wenterodt, A new approach to understanding and modelling the influence of wall roughness on friction factors for pipe and channel flows, Journal of Fluid Mechanics 613 (2008) 35 - 53. doi: $10.1017 / \mathrm{S} 0022112008003534$

[19] H. Herwig, B. Schmandt, Determination of conduit component loss coefficients based on the second law of thermodynamics, in: 23rd International Symposium on Transport Phenomena, New Zealand, 2012.

[20] H. Herwig, B. Schmandt, Loss coefficients for compressible flows in conduit components under different thermal boundary conditions, in: Proceedings 
of the 15th International Heat Transfer Conference, IHTC-15. August 1015, 2014, Kyoto, Japan, 2014.

[21] R. W. Jeppson, Steady Flow Analysis of Pipe Networks: An Instructional Manual, 1974, Reports:Paper 300.

URL https://digitalcommons.usu.edu/water_rep/300

[22] D. Brkić, Iterative methods for looped network pipeline calculation, Water Resources Management 25 (12) (2011) 2951-2987. doi:10.1007/ s11269-011-9784-3.

URL https://doi .org/10.1007/s11269-011-9784-3

[23] J. H. Manton, Differential calculus, tensor products and the importance of notation, arXiv.

URL https://arxiv.org/pdf/1208.0197.pdf

[24] I. E. Idelchik, Hanbook of hydraulic resistance (2nd Edition), Hemisphere Publishing Corporation, 1986, ISBN: 0-89116-284-4.

[25] J. Jiménez, Coherent structures in wall-bounded turbulence, Journal of Fluid Mechanics 842 (2018) P1. doi:10.1017/jfm.2018.144.

[26] L. Moody, Friction factors for pipe flow, Transactions ASME 66 (1944) $671-684$.

[27] J. McGovern, Technical note: Friction diagrams for pipe flow. dublin institute of technology.

URL https://arrow.dit.ie/engschmecart/28/(accessedJuly2018)

[28] J. Nikuradse, Strmungsgesetze in rauhen Rohren, 1933.

[29] T. M. Adams, Improved method for converting equivalent sandgrain roughness to hazen-williams coefficient, Proceedings of the 2 nd World Congress on Mechanical, Chemical, and Material Engineering (MCM'16),Budapest, Hungary doi:DOI : 10.11159/htff16.119. 
[30] K. A. Flack, M. P. Schultz, J. M. Barros, Y. C. Kim, Skin-friction behaviour in the transitionally-rough regime, International Symposium On Turbulence and Shear Flow Phenomena (TSFP-9), Melbourne Australia.

[31] J. Jimenez, Turbulent flows over rough walls, Annual Reviews of Fluid Mechanics 36 (2004) 173-196. doi:10.1146/annurev.fluid.36.050802. 122103 .

[32] N. Afzal, Friction factor directly from transitional roughness in a turbulent pipe flow, Journal of Fluids Engineering 129 (10) (2007) 1255-1267. doi: $10.1115 / 1.2776961$

[33] B. Cory, Pressure against flowrate- is the square law true?, in: AMCA International Engineering Conference (Las Vegas, NV, USA), 2008, engineering paper: 5250-08.

[34] L. Martyushev, Some interesting consequences of the maximum entropy production principle, Journal of Experimental and Theoretical Physics 104 (4) (2007) 651-654. doi:10.1134/S1063776107040152.

\section{Nomenclature}

\section{Acronyms}

$\bar{v}$ Mean velocity $\left[m \cdot s^{-1}\right.$

$\dot{Q}$ Heat flow $[W]$

$\dot{V}$ Volume flow rate $\left[m^{3} \cdot s^{-1}\right]$

$\dot{W}$ Work $[W]$

$\mathbb{D}$ Total derivative

$\widehat{K}$ Loss coefficient $\left[\mathrm{J} \cdot \mathrm{m}^{-3}\right] \equiv[P a]$

$D$ Diameter $[m]$ 
$e_{m}$ Mechanical energy (pressure,kinetic and potential) per unit mass $\left[J \cdot k g^{-1}\right]$

$g$ Gravity acceleration $\left[m \cdot s^{-2}\right]$

$H$ Head $[m]$

$p$ Pressure $[\mathrm{Pa}]$

$R e_{D}$ Reynolds number

$R e_{\phi}$ Roughness Reynolds number

$u$ Specific internal energy $\left[J \cdot k g^{-1}\right]$

$z$ Height $[m]$

\section{Greek Symbols}

$\alpha$ Correction factor for computing the kinetic energy

$\phi$ Roughness scale

$\rho$ Density $\left[k g \cdot \mathrm{m}^{-3}\right]$

$\varphi$ Energy dissipation per unit mass $\left[J \cdot k g^{-1}\right]$

$\widehat{\varphi}$ Energy dissipation per unit volume $\left[J \cdot m^{-3}\right]$ 
* computing the steady-state distribution of a flow network based on MinEP

* only dissipative characteristics of the conduit elements are needed

$*$ it is applied to any tree-shaped air flow network common in HVAC systems

$*$ a fixed point value problem emerges along with the minimisation problem 